\title{
Planck early results. XVII. Origin of the submillimetre excess dust emission in the Magellanic Clouds ${ }^{\star}$
}

Planck Collaboration: P. A. R. Ade ${ }^{73}$, N. Aghanim $^{48}$, M. Arnaud ${ }^{59}$, M. Ashdown ${ }^{57,4}$, J. Aumont ${ }^{48}$, C. Baccigalupi ${ }^{71}$, A. Balbi ${ }^{30}$, A. J. Banday ${ }^{77,7,64}$, R. B. Barreiro ${ }^{54}$, J. G. Bartlett ${ }^{3,55}$, E. Battaner ${ }^{79}$, K. Benabed ${ }^{49}$, A. Benoît ${ }^{47}$, J.-P. Bernard ${ }^{77,7}$, M. Bersanellii 27,42 , R. Bhatia ${ }^{5}$, J. J. Bock ${ }^{55,8}$, A. Bonaldi ${ }^{38}$, J. R. Bond ${ }^{6}$, J. Borrill ${ }^{63,74}$, C. Bot ${ }^{69}$, F. R. Bouchet ${ }^{49}$, F. Boulanger ${ }^{48}$, M. Bucher ${ }^{3}$, C. Burigana ${ }^{41}$, P. Cabella ${ }^{30}$, J.-F. Cardoso ${ }^{60,3,49}$, A. Catalano ${ }^{3,58}$, L. Cayón ${ }^{20}$, A. Challinor ${ }^{51,57,9}$, A. Chamballu ${ }^{45}$, L.-Y Chiang ${ }^{50}$, C. Chiang ${ }^{19}$, P. R. Christensen ${ }^{67,31}$, D. L. Clements ${ }^{45}$, S. Colombi ${ }^{49}$, F. Couchot ${ }^{62}$, A. Coulais ${ }^{58}$, B. P. Crill $^{55,68}$, F. Cuttaia ${ }^{41}$, L. Danese ${ }^{71}$, R. D. Davies ${ }^{56}$, R. J. Davis ${ }^{56}$, P. de Bernardis ${ }^{26}$, G. de Gasperis ${ }^{30}$, A. de Rosa ${ }^{41}$, G. de Zotti ${ }^{38,71}$, J. Delabrouille ${ }^{3}$, J.-M. Delouis ${ }^{49}$, F.-X. Désert ${ }^{44}$, C. Dickinson ${ }^{56}$, K. Dobashi ${ }^{14}$, S. Donzelli42,52, O. Doré5 ${ }^{55,8}$, U. Dörl ${ }^{64}$, M. Douspis ${ }^{48}$, X. Dupac ${ }^{34}$, G. Efstathiou ${ }^{51}$,

T. A. Enßlin ${ }^{64}$, F. Finelli ${ }^{41}$, O. Forni ${ }^{77,7}$, M. Frailis ${ }^{40}$, E. Franceschi ${ }^{41}$, Y. Fukui ${ }^{18}$, S. Galeotta ${ }^{40}$, K. Ganga ${ }^{3,46}$, M. Giard ${ }^{77,7}$, G. Giardino ${ }^{35}$, Y. Giraud-Héraud ${ }^{3}$, J. González-Nuevo ${ }^{71}$, K. M. Górski ${ }^{55,81}$, S. Gratton ${ }^{57,51}$, A. Gregorio ${ }^{28}$, A. Gruppuso ${ }^{41}$, D. Harrison ${ }^{51,57}$, G. Helou ${ }^{8}$, S. Henrot-Versillé ${ }^{62}$, D. Herranz ${ }^{54}$, S. R. Hildebrandt ${ }^{8,61,53}$,

E. Hivon ${ }^{49}$, M. Hobson ${ }^{4}$, W. A. Holmes ${ }^{55}$, W. Hovest ${ }^{64}$, R. J. Hoyland ${ }^{53}$, K. M. Huffenberger ${ }^{80}$, A. H. Jaffe ${ }^{45}$,

W. C. Jones ${ }^{19}$, M. Juvela ${ }^{17}$, A. Kawamura ${ }^{18}$, E. Keihänen ${ }^{17}$, R. Keskitalo ${ }^{55,17}$, T. S. Kisner ${ }^{63}$, R. Kneiss ${ }^{33,5}$, L. Knox ${ }^{22}$, H. Kurki-Suonio ${ }^{17,36}$, G. Lagache ${ }^{48}$, A. Lähteenmäki ${ }^{1,36}$, J.-M. Lamarre ${ }^{58}$, A. Lasenby ${ }^{4,57}$, R. J. Laureijs ${ }^{35}$, C. R. Lawrence ${ }^{55}$, S. Leach ${ }^{71}$, R. Leonardi ${ }^{34,35,23}$, C. Leroy ${ }^{48,77,7}$, M. Linden-Vørnle ${ }^{11}$, M. López-Caniego ${ }^{54}$, P. M. Lubin ${ }^{23}$, J. F. Macías-Pérez ${ }^{61}$, C. J. MacTavish ${ }^{57}$, S. Madden $^{59}$, B. Maffei ${ }^{56}$, N. Mandolesi ${ }^{41}$, R. Mann ${ }^{72}$, M. Maris ${ }^{40}$, E. Martínez-González ${ }^{54}$, S. Masi ${ }^{26}$, S. Matarrese ${ }^{25}$, F. Matthai ${ }^{64}$, P. Mazzotta ${ }^{30}$, P. R. Meinhold ${ }^{23}$,

A. Melchiorri ${ }^{26}$, L. Mendes ${ }^{34}$, A. Mennella 27,40 , M.-A. Miville-Deschênes 48,6 , A. Moneti ${ }^{49}$, L. Montier ${ }^{77,7}$,

G. Morgante $^{41}$, D. Mortlock ${ }^{45}$, D. Munshi ${ }^{73,51}$, A. Murphy ${ }^{66}$, P. Naselsky ${ }^{67,31}$, F. Nati ${ }^{26}$, P. Natoli ${ }^{29,2,41}$,

C. B. Netterfield ${ }^{13}$, H. U. Nørgaard-Nielsen ${ }^{11}$, F. Noviello ${ }^{48}$, D. Novikov ${ }^{45}$, I. Novikov ${ }^{67}$, T. Onishi ${ }^{15}$, S. Osborne ${ }^{76}$,

F. Pajot ${ }^{48}$, R. Paladini ${ }^{75,8}$, D. Paradis ${ }^{77,7}$, F. Pasian ${ }^{40}$, G. Patanchon ${ }^{3}$, O. Perdereau ${ }^{62}$, L. Perotto ${ }^{61}$, F. Perrotta ${ }^{71}$,

F. Piacentini ${ }^{26}$, M. Piat ${ }^{3}$, S. Plaszczynski ${ }^{62}$, E. Pointecouteau ${ }^{77,7}$, G. Polenta ${ }^{2,39}$, N. Ponthieu ${ }^{48}$, T. Poutanen ${ }^{36,17,1}$, G. Prézeau ${ }^{8,55}$, S. Prunet ${ }^{49}$, J.-L. Puget ${ }^{48}$, W. T. Reach ${ }^{78}$, R. Rebolo ${ }^{53,32}$, M. Reinecke ${ }^{64}$, C. Renault ${ }^{61}$, S. Ricciardi ${ }^{41}$, T. Riller ${ }^{64}$, I. Ristorcelli 77,7 , G. Rocha ${ }^{55,8}$, C. Rosset $^{3}$, M. Rowan-Robinson ${ }^{45}$, J. A. Rubiño-Martín ${ }^{53,32}$, B. Rusholme ${ }^{46}$, M. Sandri ${ }^{41}$, G. Savini ${ }^{70}$, D. Scott ${ }^{16}$, M. D. Seiffert ${ }^{55,8}$, G. F. Smoot ${ }^{21,63,3}$, J.-L. Starck ${ }^{59,10}$, F. Stivoli ${ }^{43}$, V. Stolyarov ${ }^{4}$, R. Sudiwala ${ }^{73}$, J.-F. Sygnet ${ }^{49}$, J. A. Tauber ${ }^{35}$, L. Terenzi ${ }^{41}$, L. Toffolatti ${ }^{12}$, M. Tomasi ${ }^{27,42}$, J.-P. Torre ${ }^{48}$, M. Tristram ${ }^{62}$, J. Tuovinen ${ }^{65}$, G. Umana ${ }^{37}$, L. Valenziano ${ }^{41}$, J. Varis ${ }^{65}$, P. Vielva ${ }^{54}$, F. Villa ${ }^{41}$, N. Vittorio ${ }^{30}$, L. A. Wade ${ }^{55}$, B. D. Wandelt ${ }^{49,24}$, A. Wilkinson ${ }^{56}$, N. Ysard ${ }^{17}$, D. Yvon ${ }^{10}$, A. Zacchei ${ }^{40}$, and A. Zonca ${ }^{23}$

(Affiliations can be found after the references)

Received 8 January 2011 / Accepted 31 May 2011

\section{ABSTRACT}

The integrated spectral energy distributions (SED) of the Large Magellanic Cloud (LMC) and Small Magellanic Cloud (SMC) appear significantly flatter than expected from dust models based on their far-infrared and radio emission. The still unexplained origin of this millimetre excess is investigated here using the Planck data. The integrated SED of the two galaxies before subtraction of the foreground (Milky Way) and background (CMB fluctuations) emission are in good agreement with previous determinations, confirming the presence of the millimetre excess. In the context of this preliminary analysis we do not propose a full multi-component fitting of the data, but instead subtract contributions unrelated to the galaxies and to dust emission.

The background CMB contribution is subtracted using an internal linear combination (ILC) method performed locally around the galaxies. The foreground emission from the Milky Way is subtracted as a Galactic H I template, and the dust emissivity is derived in a region surrounding the two galaxies and dominated by Milky Way emission. After subtraction, the remaining emission of both galaxies correlates closely with the atomic and molecular gas emission of the LMC and SMC. The millimetre excess in the LMC can be explained by CMB fluctuations, but a significant excess is still present in the SMC SED. The Planck and IRAS-IRIS data at $100 \mu \mathrm{m}$ are combined to produce thermal dust temperature and optical depth maps of the two galaxies.

^ Corresponding author: J.-P. Bernard,

e-mail: jean-philippe.bernard@cesr.fr 
The LMC temperature map shows the presence of a warm inner arm already found with the Spitzer data, but which also shows the existence of a previously unidentified cold outer arm. Several cold regions are found along this arm, some of which are associated with known molecular clouds. The dust optical depth maps are used to constrain the thermal dust emissivity power-law index $(\beta)$. The average spectral index is found to be consistent with $\beta=1.5$ and $\beta=1.2$ below $500 \mu \mathrm{m}$ for the LMC and SMC respectively, significantly flatter than the values observed in the Milky Way. Also, there is evidence in the SMC of a further flattening of the SED in the sub-mm, unlike for the LMC where the SED remains consistent with $\beta=1.5$. The spatial distribution of the millimetre dust excess in the SMC follows the gas and thermal dust distribution. Different models are explored in order to fit the dust emission in the SMC. It is concluded that the millimetre excess is unlikely to be caused by very cold dust emission and that it could be due to a combination of spinning dust emission and thermal dust emission by more amorphous dust grains than those present in our Galaxy.

Key words. Magellanic Clouds - dust, extinction - ISM: structure - galaxies: ISM - infrared: galaxies - submillimeter: galaxies

\section{Introduction}

Star formation and the exchange and evolution of materials between the stars and the interstellar medium (ISM) are continuous processes that drive the evolution of galaxies. As stars evolve, die, and renew the life cycle of dust and gas, the amount of dust and its distribution in a galaxy has important consequences for its subsequent star formation. Knowing the dust content throughout cosmic history would thus provide clues to the star formation history of the universe as the metallicities evolve. One of the puzzling results that has emerged from the studies of dust SEDs in the early universe is finding very large dust masses in high-redshift galaxies. For example, recent Herschel observations of submillimetre galaxies (SMGs) find excessively large dust masses, and high dust-to-gas mass ratios (D/G) (Santini et al. 2010). These findings contradict the low metallicities measured in the gas. Large dust masses have also been measured for a range of low-metallicity, local universe dwarf galaxies (Dumke et al. 2003; Galliano et al. 2003, 2005; Bendo et al. 2006; Galametz et al. 2009; Grossi et al. 2010; O’Halloran et al. 2010). Our understanding of how dust masses are estimated and what observational contraints are necessary is called into question by the discovery that the dust masses measured in these galaxies appear significantly larger than expected from their metal content.

Excessively large dust masses only seem to be found in low metallicity galaxies to date. The evidence is found in the behaviour of the submillimetre (submm) emission, beyond about $400-500 \mu \mathrm{m}$. From a study of a broad range of metal-rich and metal-poor galaxies, observed with wide wavelength coverage that included submm observations beyond $500 \mu \mathrm{m}$, a submm excess, beyond the normal dust SED models, was found only for low metallicity systems (Galametz et al. 2010). Such a submm excess is particularly evident in the Magellanic Clouds (Israel et al. 2010; Bot et al. 2010b). The origin of such an excess is still uncertain but several suggestions have been put forward:

- The submm excess has been modelled as a cold dust component with a submm emissivity index $(\beta)$ of $\beta=1$, which suggests a low dust temperature of $\sim 10 \mathrm{~K}$ (Galliano et al. 2003, 2005; Galametz et al. 2009, 2010). There are few observational constraints in the submm-wavelength window, hence this cold component is a rather ad hoc solution. This description often results in discrepantly large $\mathrm{D} / \mathrm{G}$ ratios being found for the low metallicity galaxies if the estimates of the total gas reservoir are well known.

- Meny et al. (2007) show that emission by amorphous grains is expected to produce excess emission in the submm wavelength range. As a result, the spectral shape of emission by amorphous grains cannot be reproduced by a single emissivity index over the whole far-infrared (FIR) to millimetre $(\mathrm{mm})$ range, and is expected to flatten at long wavelengths. The intensity of the excess is also predicted to depend strongly on the temperature of the dust grains. Modifying the optical properties of the dust to incorporate the effects of the disordered structure of the amorphous grains shows that the effective submm emissivity index decreases (thus flattening the submm Rayleigh-Jeans slope) as the dust temperature increases. Similarly, an inverse correlation between the dust temperature and the spectral index has been observed in data from the FIR to the submm (e.g. Dupac et al. 2003; Paradis et al. 2010). However, these authors, as well as Shetty et al. (2009a), advise caution in the interpretation of the observed inverse $T-\beta$ relationship, which is affected by the natural correlation between these two parameters, and requires careful treatment of observational uncertainties. Shetty et al. (2009b) also studied the effect of temperature variations along the line-of-sight on the observed $T-\beta$ relation. Paradis et al. (2009), using the DIRBE, Archeops and WMAP data, showed that the FIRsubmm SEDs of galactic molecular clouds and their neutral surroundings do indeed show a flattening beyond $\lambda \simeq$ $500 \mu \mathrm{m}$.

- Spinning dust emitted in the ionised gas of galaxies has been suggested as the explanation for the radio emission often seen in galaxies (Ferrara \& Dettmar 1994). This idea was further explored by Anderson \& Watson (1993) and Draine \& Lazarian (1998b,a), who characterised the 10 to $100 \mathrm{GHz}$ anomalous foreground emission component as radiation from very small spinning dust particles such as polycyclic aromatic hydrocarbons (PAHs). Recent improvements to the model suggest that the peak frequency, which could occur in the submm, depends on many parameters, including the radiation field intensity, the dust size distribution, the dipole moment distribution, and the physical parameters of the gas phases (Hoang et al. 2010; Silsbee et al. 2011; Ysard et al. 2010; Ysard \& Verstraete 2010). Spinning dust has been a preferred explanation for the submm excess observed on the global scale in the Magellanic Clouds (Israel et al. 2010; Bot et al. 2010b).

- Serra Díaz-Cano \& Jones (2008) have suggested hydrogenated amorphous carbon as the most likely carbonaceous grain material, rather than graphite. The advantage of using amorphous carbon, particularly in the cases where a submm excess is found, is that amorphous carbons are more emissive and result in a flatter submm slope and smaller dust mass. This has been used with new Herschel observations to model some low metallicity galaxies (Galametz et al. 2010; O'Halloran et al. 2008; Meixner et al. 2010).

- It has also been suggested that abundance increase of the hot, very small, stochastically heated grains with a low dust emissivity could be the cause of the submm emission when there is a submm excess present (Lisenfeld et al. 2001; Zhu et al. 2009).

Some of the possible causes of the submm excess could be ruled out or constrained if the gas mass estimates were better established. However, estimates of the total gas reservoirs in low 
metallicity environments have been uncertain, particularly the molecular gas component. Observations of $\mathrm{CO}$ in low metallicity galaxies have been a challenge, and the dearth of detected $\mathrm{CO}$ has often been interpreted as meaning there is very little mass in molecular gas present in galaxies which are otherwise actively forming massive stars (Leroy et al. 2009). However, relying on $\mathrm{CO}$ alone to trace the $\mathrm{H}_{2}$ mass could potentially miss a large reservoir of molecular gas residing outside the COemitting region, as indicated by the FIR fine structure observations in the Magellanic Clouds and other low metallicity galaxies (e.g. Poglitsch et al. 1995; Madden et al. 1997; Madden 2000). The presence of a hidden gas mass in low metallicity galaxies is also suggested by dust observations of the Magellanic Clouds (e.g. Bernard et al. 2008; Dobashi et al. 2008; Leroy et al. 2007; Roman-Duval et al. 2010; Bot et al. 2010a).

Most of these studies which find a submm excess in lowmetallicity galaxies are derived from models on global galaxy scales, and use data with a wavelength coverage that is limited to $\lambda<500 \mu \mathrm{m}$ with Herschel or to $\lambda<870 \mu \mathrm{m}$ for ground-based observations. The Galametz et al. (2010) study demonstrated that dust masses can differ by an order of magnitude depending on the availability of submm constraints on the SED modelling. These require wider wavelength coverage of the submm and millimetre $(\mathrm{mm})$ region and better spatial information, to map out the submm excess and determine the local physical conditions.

The closest low-metallicity galaxies are our neighbouring Large Magellanic Cloud (LMC) and Small Magellanic Cloud (SMC), which have metallicities of $50 \%$ and $20 \%$ solar metallicity $\left(Z_{\odot}\right)$ respectively. They are ideal laboratories for studying the ISM and star formation in different low metallicity environments. Recent studies with Spitzer (SAGE: Meixner et al. 2006; Bolatto et al. 2007) and Herschel (HERITAGE: Meixner et al. 2010) have mapped out the the temperature and dust mass distribution (Bernard et al. 2008; Gordon et al. 2009, 2010; Leroy et al. 2007). Recent Herschel studies of the LMC already point to a possible submm band excess and an excessive dust mass, which may be suggesting the presence of molecular gas not probed by CO. This could be explained by the presence of amorphous carbon grains (Roman-Duval et al. 2010; Meixner et al. 2010; Gordon et al. 2010). It was difficult to be conclusive about the cause of the excess seen in the Herschel observation because of the lack of longer wavelength coverage. Global excess mm and submm emission in the LMC and SMC was indisputably shown by Bot et al. (2010b) and Israel et al. (2010), using broader wavelength coverage that included the submm to cm observations of the Top-Hat balloon experiment, WMAP and COBE. Their results favour spinning dust as the explanation for the submm and mm excess, but require additional spatial coverage to be conclusive. Planck ${ }^{1}$ observations have both wide wavelength coverage and the spatial resolution to locate the submm emission within the LMC and SMC, which will help determine the origin of the submm excess. In the framework of the preliminary analysis carried out here, we focus our interest on dust emission from the galaxies themselves, which we isolate by subtracting other unrelated components, instead of carrying out a full multi-component analysis exploiting all spatial information available in the data.

\footnotetext{
1 Planck (http://www.esa.int/Planck) is a project of the European Space Agency (ESA) with instruments provided by two scientific consortia funded by ESA member states (in particular the lead countries: France and Italy) with contributions from NASA (USA), and telescope reflectors provided in a collaboration between ESA and a scientific consortium led and funded by Denmark.
}

Table 1. Characteristics of the FIR/submm data used in this study.

\begin{tabular}{llllll}
\hline \hline Data & $\begin{array}{l}\lambda_{\text {ref }} \\
{[\mu \mathrm{m}]}\end{array}$ & $\begin{array}{l}v_{\text {ref }} \\
{[\mathrm{GHz}]}\end{array}$ & $\begin{array}{l}\theta \\
{[\operatorname{arcmin}]}\end{array}$ & $\begin{array}{l}\sigma_{\mathrm{II}} \\
{\left[\mathrm{MJy} \mathrm{sr}^{-1}\right]}\end{array}$ & $\begin{array}{l}\sigma_{\text {abs }} \\
{[\%]}\end{array}$ \\
\hline IRAS & 100 & 2997.92 & 4.30 & 0.06 & $13.6^{\dagger \ddagger}$ \\
HFI & 349.82 & 857 & 3.67 & $0.12^{b}$ & $7 \%$ \\
HFI & 550.08 & 545 & 3.80 & $0.12^{b}$ & $7 \%$ \\
HFI & 849.27 & 353 & 4.43 & $0.08^{b}$ & $\lesssim 2 \%$ \\
HFI & 1381.5 & 217 & 4.68 & $0.08^{b}$ & $\lesssim 2 \%$ \\
HFI & 2096.4 & 143 & 7.04 & $0.08^{b}$ & $\lesssim 2 \%$ \\
HFI & 2997.9 & 100 & 9.37 & $0.07^{b}$ & $\lesssim 2 \%$ \\
LFI & 4285.7 & 70.3 & 13.01 & - & 5.0 \\
LFI & 6818.2 & 44.1 & 27.92 & - & 5.0 \\
LFI & 10000 & 28.5 & 32.65 & - & 5.0 \\
WMAP & 3200 & 93.69 & 13.2 & $1.76^{\sharp}$ & 1.0 \\
WMAP & 4900 & 61.18 & 21.0 & $0.36^{\sharp}$ & 1.0 \\
WMAP & 7300 & 41.07 & 30.6 & $0.11^{\sharp}$ & 1.0 \\
WMAP & 9100 & 32.94 & 39.6 & $0.05^{\sharp}$ & 1.0 \\
WMAP & 13000 & 23.06 & 52.8 & $0.02^{\sharp}$ & 1.0 \\
\hline
\end{tabular}

Notes. ${ }^{(\dagger)}$ Assumed to be for the average IRAS coverage. $\sigma_{\text {II }}$ computed by rescaling this value to actual coverage. ${ }^{(\ddagger)}$ From Miville-Deschênes \& Lagache (2005). ${ }^{(b)} 1 \sigma$ average value in one beam scaled from Planck HFI Core Team (2011b). We actually use the internal variance maps for $\sigma_{\mathrm{II}}{ }^{(\sharp)}$ From Bennett et al. (2003).

\section{Observations}

\subsection{Planck data}

The Planck first mission results are presented in Planck Collaboration (2011a) and the in-flight performances of the two focal plane instruments, the HFI (High Frequency Instrument) and the LFI (Low Frequency Instrument), are given in Planck HFI Core Team (2011a) and Mennella et al. (2011) respectively. The data processing and calibration of the HFI and LFI data used here is described in Planck HFI Core Team (2011b) and Zacchei et al. (2011) respectively.

Figure 1 shows the total intensity maps, observed toward the LMC and SMC at three HFI and LFI frequencies. Both galaxies are well detected at high frequencies. Around $100 \mathrm{GHz}$, their emission can barely be distinguished from CMB fluctuations. At lower frequencies, the contrast between the galaxies' emission and the CMB fluctuations becomes larger again. Note that the apparent variation of the noise level at a constant right-ascension value across the LMC is real and is due to the LMC being positioned at the edge of the Planck deep field.

The Planck DR2 data have had the CMB fluctuations removed in a way which is inappropriate for detailed examination of foreground sources like the LMC and SMC. We therefore use the original data before CMB subtraction (version DX4 of the Planck-HFI and Planck-LFI data) and perform our own subtraction of the CMB fluctuations, based on a local ILC (see Sect. 2.3.2).

We use the internal variance $\left(\sigma_{\mathrm{II}}^{2}\right)$ provided with the Planck data, which represents the white noise on the intensity. The LMC and SMC have been observed many times, as the Planck scanning strategy (Planck Collaboration 2011a) covers the region close to the ecliptic pole repeatedly. In particular, the LMC is located at the boundary of the Planck deep field. Its eastern half has a much higher signal to noise ratio than its western half. We assume the absolute uncertainties from calibration given in Planck HFI Core Team (2011b) and Zacchei et al. (2011) for HFI and LFI respectively and summarised in Table 1. 

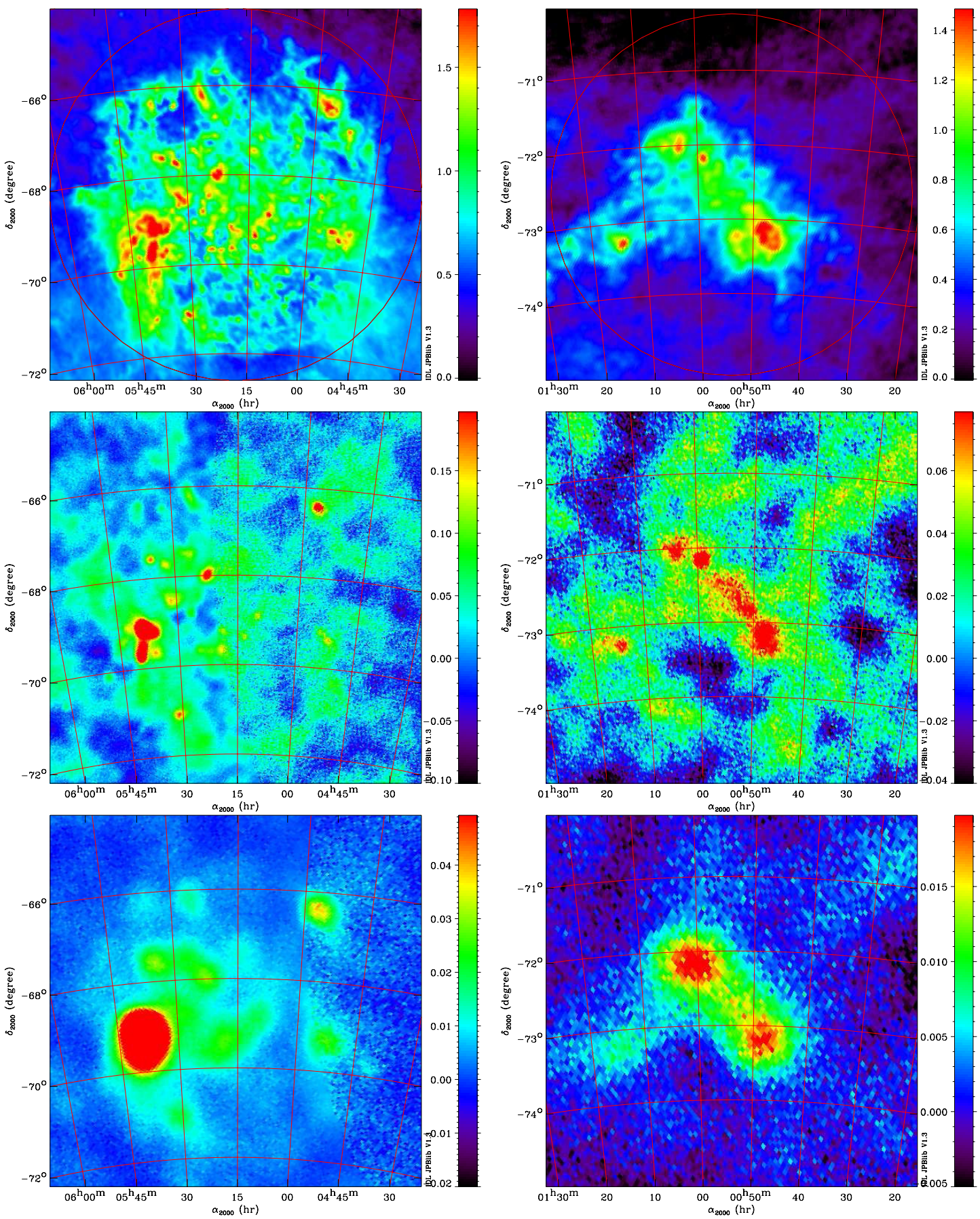

Fig. 1. Planck total intensity data for the LMC (left) and SMC (right) at 857 (top), 100 (middle) and $28.5 \mathrm{GHz}($ bottom) at full resolution. The top panels are shown in log scale. The circle in the top panels shows the region used to extract average SEDs.

\subsection{Ancillary data}

\subsubsection{HI LMC/SMC data}

To trace the atomic gas in the Magellanic Clouds, we used H I maps in the $21 \mathrm{~cm}$ line, obtained by combining data from the Australia Telescope Compact Array (ATCA) and the Parkes single dish telescope. For the LMC, this data was obtained by Kim et al. (2003) and Staveley-Smith et al. (2003) and covers $11.1^{\circ} \times 12.4^{\circ}$ on the sky. The spatial resolution is $1^{\prime}$, corresponding to a physical resolution of about $14.5 \mathrm{pc}$ at the distance of the LMC. For the SMC, the data were obtained by Staveley-Smith et al. (1997) and Stanimirovic et al. (1999). The area covered is a $4.5^{\circ} \times 4.5^{\circ}$ region. Hi observations of the SMC tail $\left(7^{\circ} \times 6^{\circ}\right)$ were obtained by Muller et al. (2003) and combined with the observations taken in the direction of the SMC. The spatial resolution is $98^{\prime \prime}$, corresponding to $30 \mathrm{pc}$ at the distance of the SMC.

\subsubsection{H। Galactic data}

The LMC and SMC are located at galactic latitudes $b_{\mathrm{II}}=-34^{\circ}$ and $b_{\mathrm{II}}=-44^{\circ}$ respectively. They can therefore suffer from significant contamination by Galactic foreground emission, which has to be removed from the IR data. In order to account for the Galactic foreground emission, we used Galactic H i column density maps. 
For the LMC, the $\mathrm{Hi}$ foreground map was constructed by Staveley-Smith et al. (2003) by integrating the Parkes H I data in the velocity range from $-64<v_{\text {hel }}<100 \mathrm{~km} \mathrm{~s}^{-1}$, which excludes all LMC and SMC associated gas $\left(v>100 \mathrm{~km} \mathrm{~s}^{-1}\right)$ but includes essentially all Galactic emission. The spatial resolution is $14^{\prime}$. For the SMC, a map of combined ATCA and Parkes data was build by integrating the Galactic velocities by E. Muller (priv. comm.). The resolution is $98^{\prime \prime}$.

These maps show that the Galactic foreground across the LMC is as strong as $N_{\mathrm{H}}=1.3 \times 10^{21} \mathrm{H} \mathrm{cm}^{-2}$, with significant variation across the LMC, particularly in a wide filamentary structure oriented southwest to northeast. The Galactic foreground across the SMC is weaker, with values $N_{\mathrm{H}} \simeq 3.2 \times$ $10^{20} \mathrm{H} \mathrm{cm}^{-2}$ but the associated FIR-submm emission is nonnegligible since the SMC emission is weaker than that of the LMC, due to its lower dust and gas content.

These foreground maps are used to subtract the foreground IR emission from the IR maps, using the emissivity SED described in Sect. 2.3.3.

\subsubsection{CO data}

The ${ }^{12} \mathrm{CO}(J=1 \rightarrow 0)$ molecular data used in this work was obtained using the NANTEN telescope, a 4-m radio telescope of Nagoya University at Las Campanas Observatory, Chile (see Fukui et al. 2008). The observed region covers about 30 square degrees where CO clouds were detected in the NANTEN first survey (e.g. Fukui et al. 1999; Mizuno et al. 2001; Fukui et al. 2008). The observed grid spacing was $2^{\prime}$, corresponding to about 30 and $35 \mathrm{pc}$ at the distance of the LMC and SMC, while the half-power beam width was $2.6^{\prime}$ at $115 \mathrm{GHz}$.

We used the CO maps of the Magellanic Clouds to construct an integrated intensity map $\left(W_{\mathrm{CO}}\right)$, integrating over the full $v_{\mathrm{lsr}}$ range of the data $\left(100<v_{\mathrm{lsr}}<400 \mathrm{~km} \mathrm{~s}^{-1}\right.$ for about $80 \%$ of the data, while the remaining $20 \%$ had a velocity range of about $100 \mathrm{~km} \mathrm{~s}^{-1}$ covering the $\mathrm{H}$ i emitting regions.)

\subsection{4. $\mathrm{H} \alpha$ data}

In order to estimate the free-free contribution to the millimetre fluxes, we used the continuum-subtracted $\mathrm{H}_{\alpha}$ maps from the Southern H-Alpha Sky Survey Atlas (SHASSA, Gaustad et al. 2001) centred on the Magellanic Clouds.

\subsubsection{FIR-submm data}

We used the following FIR-submm ancillary data.

- IRAS-IRIS (improved reprocessing of the IRAS survey) $100 \mu \mathrm{m}$ data, in order to constrain the dust temperature. The characteristics of these data, including the noise properties, were taken from Miville-Deschênes \& Lagache (2005) and are summarised in Table 1

- WMAP 7 yr data. The characteristics of these data, including the noise properties, were taken from Bennett et al. (2003) and Jarosik et al. (2011) and are summarised in Table 1.

\subsection{Additional data processing}

\subsubsection{Common angular resolution and pixelisation}

For data already available in the HEALPix (Górski et al. 2005) format (e.g., WMAP), we obtained the data from the Lambda web site (http://lambda.gsfc.nasa.gov/). For data not

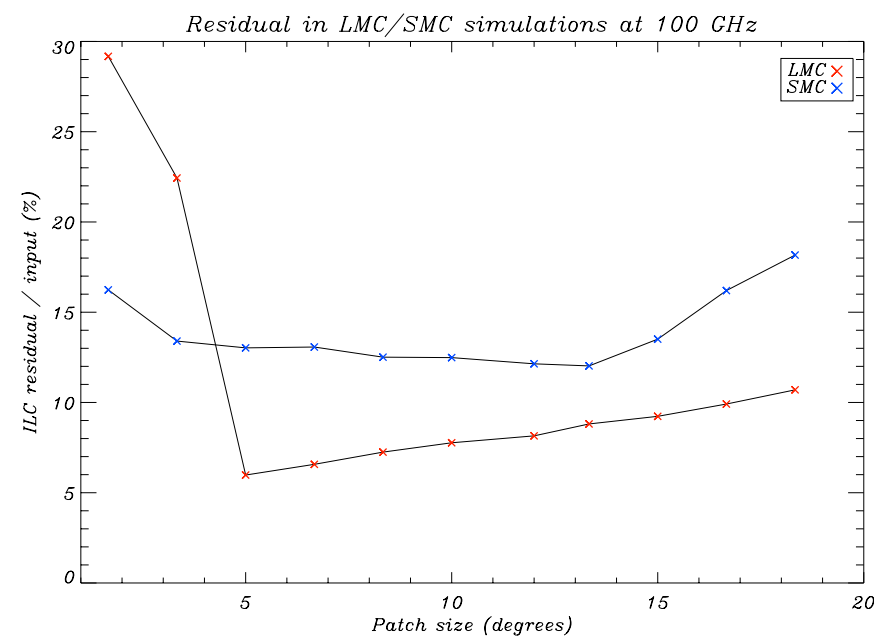

Fig. 2. Error due to the ILC CMB subtraction derived from Monte-Carlo simulations for both LMC and SMC at $100 \mathrm{GHz}$, as a function of the patch size. The values shown are the difference between the recovered and the input CMB divided by the simulated LMC and SMC, both integrated in a $4^{\circ}$ ring.

originally presented in the HEALPix format, the ancillary data were brought to the HEALPix pixelisation, using a method where the area of the surface of intersection between each HEALPix and FITS pixel of the original data was used as a weight to regrid the data. The HEALPix resolution was chosen so as to match the Shannon sampling of the original data at resolution $\theta$, with a HEALPix resolution set so that the pixel size is $<\theta / 2.4$. The ancillary data and the description of their processing will be presented in Paradis et al. (in prep.).

All ancillary data were then smoothed to an appropriate resolution by convolution with a Gaussian smoothing function with appropriate FWHM, using the smoothing HEALPix function. They were brought to a pixel size matching the Shannon sampling of the final resolution.

\subsection{2. $\mathrm{CMB}$ subtraction}

The upper panel in Fig. 3 shows the total intensity maps observed toward the LMC and SMC before CMB subtraction in the LFI 70.3 GHz chanel. The amplitude of CMB fluctuations is of the order of that of the diffuse emission of the galaxies and it is clear that, in order to study the integrated SED of the LMC and SMC galaxies, an efficient CMB subtraction has to be performed.

The standard CMB-subtracted maps produced by the data processing centre (DPC) (Planck HFI Core Team 2011b) were not used in this analysis. They were processed by a needlet internal linear combination method (NILC) (Planck HFI Core Team $2011 \mathrm{~b}$ ) that left a significant amount of foreground emission in the CMB estimate towards the LMC and the SMC.

For this reason we have subtracted an estimate of the CMB optimised locally for the LMC and SMC regions, as described below. This CMB component was reconstructed through a classical ILC by means of Lagrange multipliers (Eriksen et al. 2004). $12^{\circ} \times 12^{\circ}$ patches around the LMC and SMC were extracted from the HFI CMB frequency channels maps (100, 143, 217 and $353 \mathrm{GHz}$ ) reduced to a common resolution (10 arcmin), in units of $\mathrm{K}_{\mathrm{CMB}}$. The $\mathrm{CMB}$ component obtained on these patches clearly contains less LMC and SMC residual than the standard HFI DPC CMB and therefore will affect the SED determination less. The 

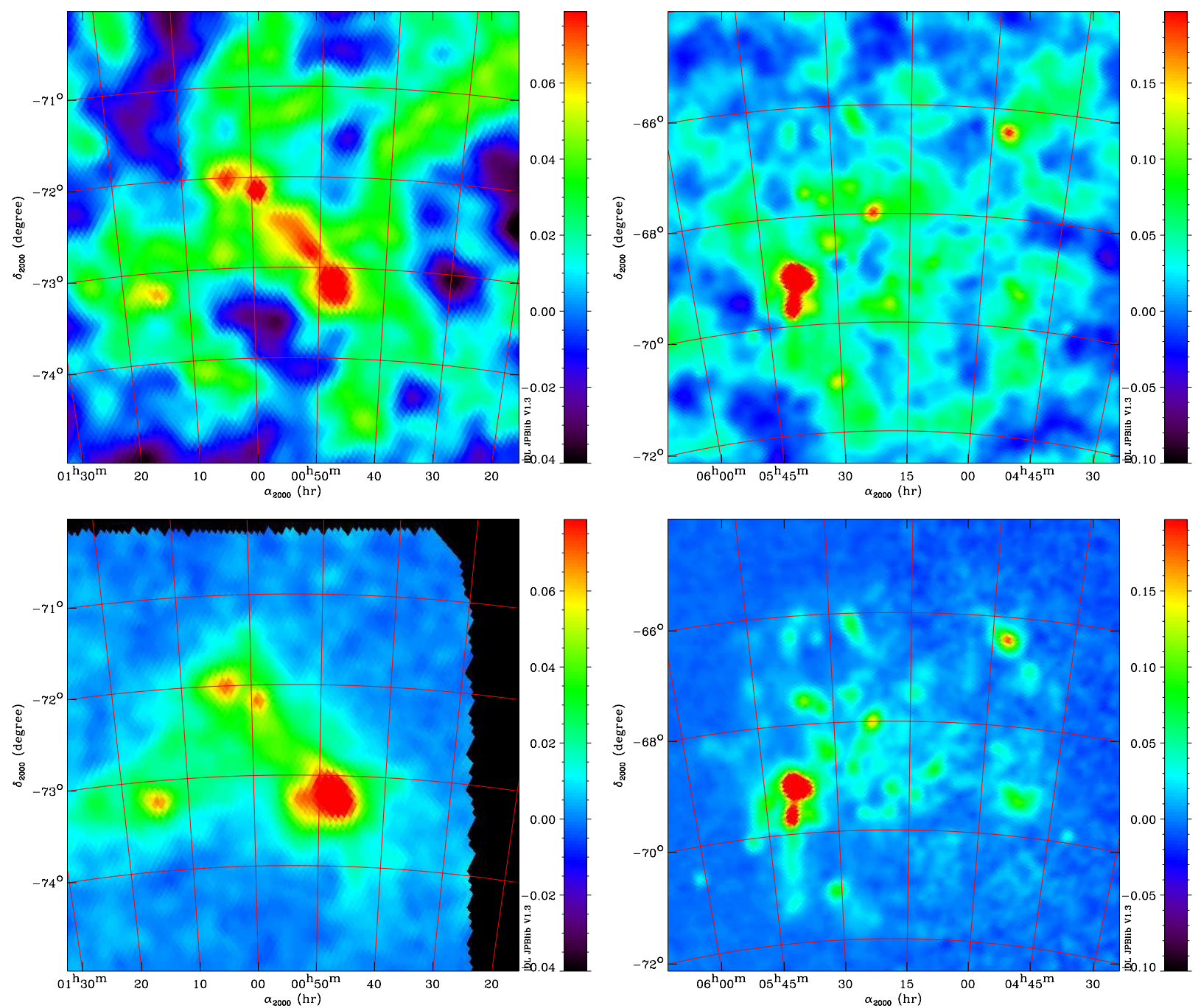

Fig. 3. SMC (left) and LMC (right) total intensity maps before (top) and after (bottom) CMB subtraction in the $70.3 \mathrm{GHz}$ band at the 13.01 arcmin resolution.

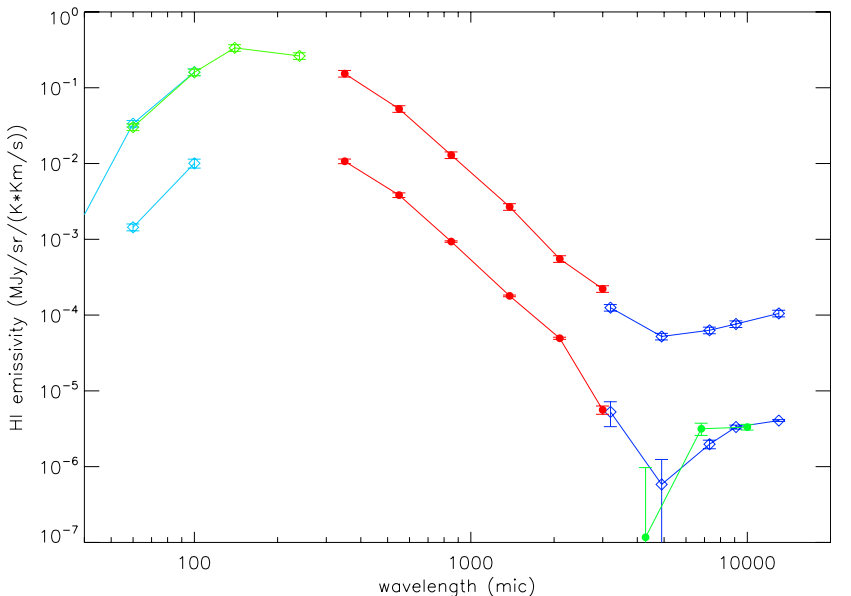

Fig. 4. Average foreground SED in the direction of the LMC and SMC (lower curve) including IRAS-IRIS (light blue), Planck-LFI (red), Planck-LFI (green) and WMAP data (dark blue), compared to the SED of the high latitude low column-density MW SED (upper curve) derived in Planck Collaboration (2011o), which has been scaled up by a factor of 10 for clarity.

lower panel in Fig. 3 shows the maps after CMB subtraction in the LFI $70.3 \mathrm{GHz}$ channel.
We performed Monte-Carlo simulations in order to estimate the error induced on the SED by our CMB removal. The LMC and SMC were simulated as a sum of two correlated components. The spatial template for the first component is the IRAS-IRIS $100 \mu \mathrm{m}$ map. The spatial template for the second component is the $545 \mathrm{GHz}$ LMC or SMC Planck map. Their correlation coefficients are $83 \%$ for the LMC and $92 \%$ for the SMC. We normalised their fluxes inside a $4^{\circ}$ ring to the value of a typical LMC or SMC dust component and a typical millimetre excess at each HFI CMB frequency, respectively. 200 independent realisations of a WMAP 7 yr best-fit CMB (Komatsu et al. 2011) and of nominal inhomogeneous HFI white noise were added to the synthetic LMC and SMC on patches of varying sizes. For each of these simulations, for each size, our ILC is performed and compared with the input CMB. We estimate the error due to the CMB subtraction in both the LMC and the SMC by comparing the residual in the CMB map to the emission of the two components at a given frequency. Results at $100 \mathrm{GHz}$ are displayed in Fig. 2. For both the LMC and the SMC, the error increases at small and large patch sizes and an optimal patch size with respect to the CMB subtraction can be found at $5^{\circ}$ for the LMC and $13^{\circ}$ for the SMC. The narrower the patch, the lower is the contribution of the $\mathrm{CMB}$ to the total variance which is to be minimised in the ILC. On the other hand, when their sizes increase, 
Planck Collaboration: Planck early results. XVII.
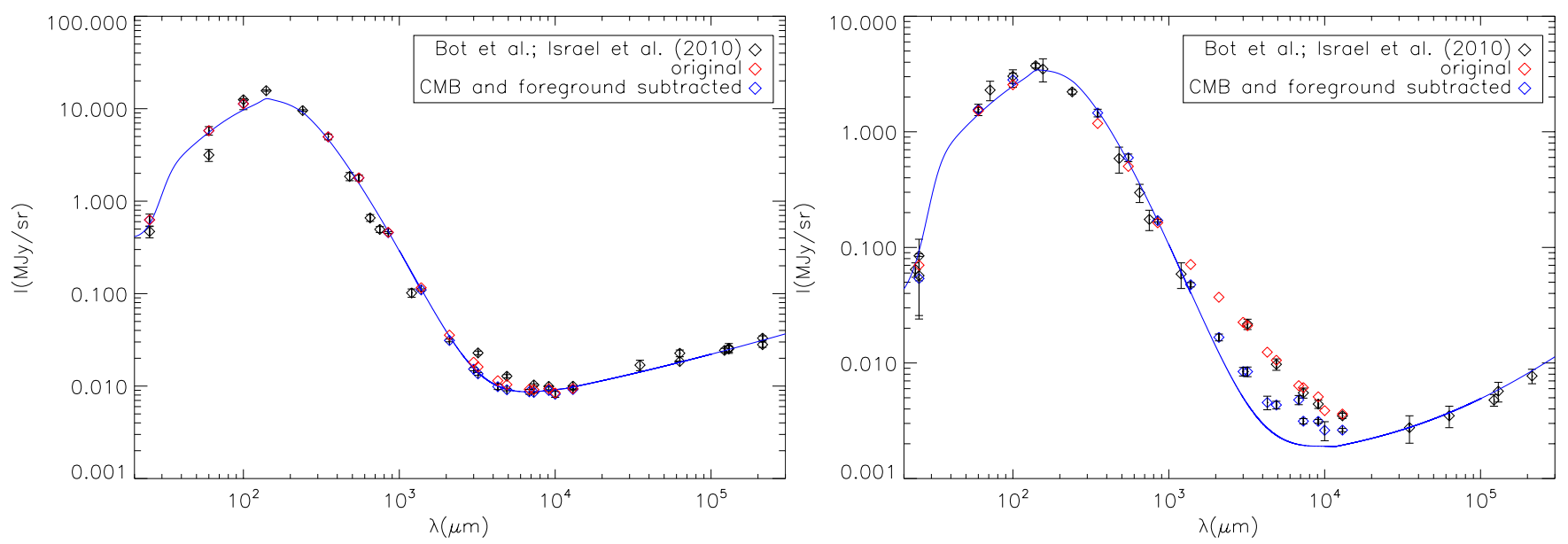

Fig. 5. Integrated SEDs of the LMC (left) and SMC (right) before and after CMB subtraction. The black points and model are taken from Bot et al. (2010b). The red symbols show the SEDs derived from the DIRBE, IRAS, and WMAP data before CMB subtraction. The blue symbols show the same after CMB subtraction.

patches include foreground emission which is uncorrelated with the galaxies and has a different spectrum, and thus both small and large patches contribute to the total variance which must be minimised.

For the $12^{\circ} \times 12^{\circ}$ patches used in the following analysis, we estimate the error due to $\mathrm{CMB}$ subtraction as $10.38 \mu K_{\mathrm{CMB}}$ and $28.2 \mu K_{\mathrm{CMB}}$ for the $\mathrm{LMC}$ and SMC respectively. In terms of the fraction of the total galaxy brightness, these correspond to 8.1, $6.2,2.3$ and $0.3 \%$ for the LMC and $12.1,10.7,6.4$ and $1.4 \%$ for the SMC at 100, 143, 217 and $353 \mathrm{GHz}$, respectively.

\subsubsection{Galactic foreground subtraction}

The Milky Way (MW) emission is non-negligible compared to the emission of the LMC and SMC. We remove this contribution at all wavelengths using the MW $\mathrm{H}_{\mathrm{I}}$ template described in Sect. 2.2.2. We first computed the correlation of all FIRsubmm data with this template, in the region where both the MW H I template and the CMB estimate are available. We excluded a circular region centred on each galaxy (centre coordinates taken as $\alpha_{2000}=05^{\mathrm{h}} 18^{\mathrm{m}} 14.8^{\mathrm{s}}, \delta_{2000}=-68^{\circ} 26^{\prime} 34.5^{\prime \prime}$ and $\alpha_{2000}=00^{\mathrm{h}} 53^{\mathrm{m}} 59.6^{\mathrm{s}}, \delta_{2000}=-72^{\circ} 40^{\prime} 16.1^{\prime \prime}$ for the LMC and $\mathrm{SMC}$ respectively) with radius $4.09^{\circ}$ and $2.38^{\circ}$ for the $\mathrm{LMC}$ and SMC respectively. The spectral distribution of this correlation factor, taken to represent the SED of the MW foreground is shown in Fig. 4. The SED is compared to that of the high galactic latitude reference region used in Planck Collaboration (2011o) in Fig. 4. It can be seen that the two SEDs are similar, although the MW foreground towards the LMC and SMC appears slightly colder and has a relatively stronger non-thermal component in the millimetre wavelength range. We subtracted the MW foreground from all the data using this SED multiplied by the MW Hi template over the full map extent. Note that the median foreground $\mathrm{H}_{\mathrm{I}}$ integrated intensities over the LMC and SMC are $\simeq 297 \mathrm{~K} \mathrm{~km} \mathrm{~s}^{-1}$ and $\simeq 172 \mathrm{~K} \mathrm{~km} \mathrm{~s}^{-1}$, which correspond to a brightnesses of $3.0 \mathrm{MJy} \mathrm{sr}^{-1}$ and $1.7 \mathrm{MJy} \mathrm{sr}^{-1}$ respectively at $857 \mathrm{GHz}$. This is of the same order as the average brightness of the galaxies at that frequency (see Table 2). However, most of the MW emission is canceled when subtracting a local background around the galaxies and the differential correction due to the spatial structure of the MW foreground then accounts for about $0.4 \%$ and $21 \%$ of the LMC and the SMC brightness respectively.

\section{Integrated SEDs}

The integrated SEDs of the LMC and SMC before and after CMB subtraction are shown in Fig. 5 (red and blue diamonds, respectively). They were computed by averaging values in the circular area around each galaxy defined in Sect. 2.3.3, and subtracting an estimate of the sky off the source taken in an annulus with radius $1^{\circ}$. They are compared to the data taken from Israel et al. (2010) and Bot et al. (2010b), which were integrated over the same region. It can be seen that the flux observed in the HFI and LFI bands is consistent with that obtained by previous studies in this wavelength range before subtraction of the CMB fluctuations. A model of thermal dust, free-free and synchrotron emission is fitted to the data as follows. As in Bot et al. (2010b), the thermal dust emission is adjusted according to the Draine \& Li (2007) dust model. The free-free emission is deduced from the $\mathrm{H}_{\alpha}$ integrated flux, using the expression from Hunt et al. (2004), assuming an electronic temperature $T_{\mathrm{e}}=10^{4} \mathrm{~K}$, the ratio of ionised helium to hydrogen $n_{\mathrm{He}^{+}} / n_{\mathrm{H}}^{+}=0.087$, and no extinction. The synchrotron emission was fitted to the radio data from the literature as in Israel et al. (2010). The combined dust, freefree and synchrotron emission is shown by the blue line in Fig. 5. It can be seen from Fig. 5 that the SED after subtraction of the CMB fluctuations is in fact compatible with no millimetre emission excess for the LMC, for this particular model. However, the CMB-subtracted SED still shows a significant excess for the SMC, with most data points above $\lambda \simeq 1 \mathrm{~mm}$ being in excess over the model by more than $5 \sigma$, leading to an overall significance of the excess of about $50 \sigma$.

The CMB subtraction removes part of the millimetre excess in both galaxies. This shows that CMB fluctuations behind the LMC and the SMC average out to a small but positive contribution when integrated over the extent of the galaxies. We note that some excess emission remains in the SMC regardless of the dust model used and the assumptions made on the free-free or synchrotron emission. The shape and intensity of the millimetre excess can change, but we could not find a solution where the SMC SED is explained purely by thermal dust emission, free-free and synchrotron radiation. We emphasise also that the dust model, used to reproduce the dust emission up to the submillimetre wavelength range, assumes components heated by a radiation field 10 times lower than the solar neighbourhood radiation field. Compared to other nearby galaxies, this result is rather extreme (Draine et al. 2007; Bot et al. 2010b). 
Table 2. LMC (Cols. 2-4) and SMC (Cols. 5-7) SEDs averaged in a circular region for each galaxy.

\begin{tabular}{|c|c|c|c|c|c|c|}
\hline $\begin{array}{l}\lambda \\
{[\mu \mathrm{m}]}\end{array}$ & $\begin{array}{c}I_{v}^{\text {tot }} \\
{\left[\mathrm{MJy} \mathrm{sr}^{-1}\right]}\end{array}$ & $\begin{array}{c}I_{v}^{\mathrm{noCMB}} \\
{\left[\mathrm{MJy} \mathrm{sr}^{-1}\right]}\end{array}$ & $\begin{array}{c}I_{v}^{\text {sub }} \\
{\left[\mathrm{MJy} \mathrm{sr}^{-1}\right]}\end{array}$ & $\begin{array}{c}I_{v}^{\mathrm{tot}} \\
{\left[\mathrm{MJy} \mathrm{sr}^{-1}\right]}\end{array}$ & $\begin{array}{c}I_{v}^{\mathrm{noCMB}} \\
{\left[\mathrm{MJy} \mathrm{sr}^{-1} \text { ] }\right.}\end{array}$ & $\begin{array}{c}I_{v}^{\text {sub }} \\
{\left[\mathrm{MJy} \mathrm{sr}^{-1}\right]}\end{array}$ \\
\hline \multicolumn{7}{|l|}{ IRAS: } \\
\hline 12 & $(2.36 \pm 0.12) \times 10^{-1}$ & $(2.36 \pm 0.12) \times 10^{-1}$ & $(2.34 \pm 0.13) \times 10^{-1}$ & $(1.37 \pm 0.11) \times 10^{-2}$ & $(1.37 \pm 0.11) \times 10^{-2}$ & $(-2.79 \pm 0.27) \times 10^{-2}$ \\
\hline 25 & $(6.28 \pm 0.95) \times 10^{-1}$ & $(6.28 \pm 0.95) \times 10^{-1}$ & $(6.25 \pm 0.96) \times 10^{-1}$ & $(7.01 \pm 1.10) \times 10^{-2}$ & $(7.01 \pm 1.10) \times 10^{-2}$ & $(5.30 \pm 0.98) \times 10^{-2}$ \\
\hline 60 & $5.79 \pm 0.60$ & $5.79 \pm 0.60$ & $5.78 \pm 0.61$ & $1.52 \pm 0.16$ & $1.52 \pm 0.16$ & $1.56 \pm 0.18$ \\
\hline 100 & $(1.13 \pm 0.15) \times 10^{1}$ & $(1.13 \pm 0.15) \times 10^{1}$ & $(1.13 \pm 0.15) \times 10^{1}$ & $2.56 \pm 0.35$ & $2.56 \pm 0.35$ & $2.82 \pm 0.39$ \\
\hline \multicolumn{7}{|c|}{ Planck: } \\
\hline 349.82 & $4.97 \pm 0.35$ & $4.97 \pm 0.35$ & $4.96 \pm 0.35$ & $1.18 \pm 0.08$ & $1.18 \pm 0.08$ & $1.46 \pm 0.11$ \\
\hline 550.08 & $1.79 \pm 0.13$ & $1.79 \pm 0.13$ & $1.78 \pm 0.13$ & $(5.02 \pm 0.37) \times 10^{-1}$ & $(4.99 \pm 0.37) \times 10^{-1}$ & $(5.96 \pm 0.45) \times 10^{-1}$ \\
\hline 849.27 & $(4.61 \pm 0.10) \times 10^{-1}$ & $(4.58 \pm 0.10) \times 10^{-1}$ & $(4.51 \pm 0.10) \times 10^{-1}$ & $(1.63 \pm 0.04) \times 10^{-1}$ & $(1.47 \pm 0.04) \times 10^{-1}$ & $(1.69 \pm 0.05) \times 10^{-1}$ \\
\hline 1381.5 & $(1.16 \pm 0.02) \times 10^{-1}$ & $(1.10 \pm 0.03) \times 10^{-1}$ & $(1.04 \pm 0.03) \times 10^{-1}$ & $(7.12 \pm 0.18) \times 10^{-2}$ & $(4.30 \pm 0.19) \times 10^{-2}$ & $(4.63 \pm 0.20) \times 10^{-2}$ \\
\hline 2096.4 & $(3.56 \pm 0.08) \times 10^{-2}$ & $(3.13 \pm 0.10) \times 10^{-2}$ & $(2.51 \pm 0.09) \times 10^{-2}$ & $(3.71 \pm 0.10) \times 10^{-2}$ & $(1.54 \pm 0.11) \times 10^{-2}$ & $(1.54 \pm 0.11) \times 10^{-2}$ \\
\hline 2997.9 & $(1.81 \pm 0.05) \times 10^{-2}$ & $(1.53 \pm 0.06) \times 10^{-2}$ & $(8.96 \pm 0.48) \times 10^{-3}$ & $(2.25 \pm 0.07) \times 10^{-2}$ & $(8.29 \pm 0.71) \times 10^{-3}$ & $(7.09 \pm 0.69) \times 10^{-3}$ \\
\hline 4285.7 & $(1.14 \pm 0.07) \times 10^{-2}$ & $(9.87 \pm 0.68) \times 10^{-3}$ & $(3.34 \pm 0.36) \times 10^{-3}$ & $(1.24 \pm 0.09) \times 10^{-2}$ & $(4.55 \pm 0.61) \times 10^{-3}$ & $(3.16 \pm 0.54) \times 10^{-3}$ \\
\hline 6818.2 & $(9.24 \pm 0.52) \times 10^{-3}$ & $(8.57 \pm 0.51) \times 10^{-3}$ & $(1.72 \pm 0.17) \times 10^{-3}$ & $(6.37 \pm 0.49) \times 10^{-3}$ & $(2.94 \pm 0.34) \times 10^{-3}$ & $(1.57 \pm 0.28) \times 10^{-3}$ \\
\hline 10000 & $(8.43 \pm 0.61) \times 10^{-3}$ & $(8.17 \pm 0.60) \times 10^{-3}$ & $(1.05 \pm 0.25) \times 10^{-3}$ & $(3.87 \pm 0.55) \times 10^{-3}$ & $(2.53 \pm 0.49) \times 10^{-3}$ & $(1.10 \pm 0.41) \times 10^{-3}$ \\
\hline \multicolumn{7}{|c|}{ WMAP: } \\
\hline 3200 & $(1.61 \pm 0.04) \times 10^{-2}$ & $(1.36 \pm 0.05) \times 10^{-2}$ & $(7.23 \pm 0.43) \times 10^{-3}$ & $(2.10 \pm 0.08) \times 10^{-2}$ & $(8.28 \pm 0.80) \times 10^{-3}$ & $(7.07 \pm 0.79) \times 10^{-3}$ \\
\hline 4900 & $(1.04 \pm 0.02) \times 10^{-2}$ & $(9.13 \pm 0.23) \times 10^{-3}$ & $(2.51 \pm 0.17) \times 10^{-3}$ & $(1.06 \pm 0.03) \times 10^{-2}$ & $(4.32 \pm 0.33) \times 10^{-3}$ & $(2.92 \pm 0.31) \times 10^{-3}$ \\
\hline 7300 & $(9.15 \pm 0.13) \times 10^{-3}$ & $(8.56 \pm 0.15) \times 10^{-3}$ & $(1.66 \pm 0.09) \times 10^{-3}$ & $(6.12 \pm 0.15) \times 10^{-3}$ & $(3.09 \pm 0.16) \times 10^{-3}$ & $(1.67 \pm 0.15) \times 10^{-3}$ \\
\hline 9100 & $(9.38 \pm 0.12) \times 10^{-3}$ & $(8.98 \pm 0.13) \times 10^{-3}$ & $(1.93 \pm 0.06) \times 10^{-3}$ & $(5.10 \pm 0.11) \times 10^{-3}$ & $(3.04 \pm 0.11) \times 10^{-3}$ & $(1.63 \pm 0.10) \times 10^{-3}$ \\
\hline 13000 & $(9.38 \pm 0.11) \times 10^{-3}$ & $(9.19 \pm 0.12) \times 10^{-3}$ & $(1.88 \pm 0.05) \times 10^{-3}$ & $(3.62 \pm 0.08) \times 10^{-3}$ & $(2.52 \pm 0.09) \times 10^{-3}$ & $(1.07 \pm 0.07) \times 10^{-3}$ \\
\hline \multicolumn{7}{|c|}{$\mathrm{H}_{\alpha}(\mathrm{R}):$} \\
\hline- & $(1.93 \pm 0.19) \times 10^{1}$ & & & $7.00 \pm 0.72$ & & \\
\hline \multicolumn{7}{|c|}{$W_{\mathrm{HI}}^{\mathrm{MW}}\left(\mathrm{K} \mathrm{km} \mathrm{s}^{-1}\right)$ : } \\
\hline- & $1.01 \pm 0.13$ & & & $(-2.57 \pm 0.26) \times$ & & \\
\hline
\end{tabular}

Notes. The integration region is centred on $\alpha_{2000}=05^{\mathrm{h}} 18^{\mathrm{m}} 14.8^{\mathrm{s}}, \delta_{2000}=-68^{\circ} 26^{\prime} 34.5^{\prime \prime}$, with radius $\mathrm{R}_{\mathrm{LMC}}=4.09^{\circ}$ for the $\mathrm{LMC}$ and centred on $\alpha_{2000}=00^{\mathrm{h}} 53^{\mathrm{m}} 59.6^{\mathrm{s}}, \delta_{2000}=-72^{\circ} 40^{\prime} 16.1^{\prime \prime}$, with radius $R_{\mathrm{SMC}}=2.38^{\circ}$ for the SMC. A common background was subtracted in a $1^{\circ}$ annulus around this region. Brightness values are in $\mathrm{MJy} \mathrm{sr}^{-1}$ in the $v \mathrm{I}_{v}=$ cste flux convention. The last two lines give the average $\mathrm{H}_{\alpha}$ emission (in Rayleigh), Galactic HI emission (in $\mathrm{K} \mathrm{km} \mathrm{s}^{-1}$ ) in the same area. The table lists the total SED $\left(I_{v}^{\mathrm{tot}}\right)$, the CMB subtracted SED $\left(I_{v}^{\mathrm{noCMB}}\right)$ and the CMB, MW foreground and free-free subtracted $\operatorname{SED}\left(I_{v}^{\text {sub }}\right)$ and their associated $1 \sigma$ uncertainties.

The integrated SEDs of the LMC and SMC after CMB and foreground subtraction are compared in Fig. 6. These SEDs were computed in the same integration region as those in Fig. 5. The comparison in Fig. 6 shows that the SMC SED is flatter than the LMC SED in the submm, while when the SEDs are normalised in the FIR, they are similar above $10 \mathrm{~mm}$, where the emission is presumably dominated by free-free and/or spinning dust (see Sect. 5).

The SEDs at various stages of the background and foreground subtraction are given in Table 2 for the LMC and SMC. The uncertainties given include the contribution from the data variance combined for the integration region, the data variance combined for the background region, and the absolute calibration uncertainties, using the values given in Table 1. They also include the noise resulting from the background (CMB) subtraction and from the foreground MW subtraction and the free-free removal. All uncertainty contributions were added quadratically.

\section{Dust temperature and emissivity}

\subsection{Temperature determination}

As shown by several previous studies (e.g. Reach et al. 1995; Finkbeiner et al. 1999; Paradis et al. 2009; Planck Collaboration $2011 \mathrm{o}, \mathrm{t})$, the dust emissivity spectrum in our Galaxy cannot be represented by a single dust emissivity index $\beta$ over the full FIR-submm domain. The data available indicate that $\beta$ is usually steeper in the FIR and flatter in the submm, with a transition around $500 \mu \mathrm{m}$ (see Paradis et al. 2009; Planck Collaboration 2011t). This is likely to be the case also for the LMC and the
SMC. The dust temperature derived will depend on the assumptions made about $\beta$, since these two parameters are somewhat degenerate in $\chi^{2}$ space. Note that some of the past attempts at constraining the equilibrium temperature of the large grains in the LMC used the IRAS $60 \mu \mathrm{m}$ emission. Emission at $60 \mu \mathrm{m}$ is highly contaminated by out-of-equilibrium emission from very small grains (VSGs) and this is even more the case in the Magellanic Clouds, due to the presence of the $70 \mu$ m excess (Bot et al. 2004; Bernard et al. 2008). Combining the IRAS $60 \mu \mathrm{m}$ and $100 \mu \mathrm{m}$ data therefore strongly over-estimates the temperature and accordingly under-estimates the abundances of all types of dust particles. A good sampling at frequencies dominated by big grain $(\mathrm{BG})$ emission became possible using the combination of the IRAS and Spitzer data (Leroy et al. 2007; Bolatto et al. 2007; Bernard et al. 2008; Sandstrom et al. 2010).

As the dust temperature is best derived from the FIR data, we limit the range of frequencies used in the determination to the FIR, which limits the impact of potential changes of the dust emissivity index $\beta$ with frequency. In the determination of the dust temperature $\left(T_{\mathrm{D}}\right)$, we used the IRAS-IRIS $100 \mu \mathrm{m}$ map and the two highest HFI frequencies at 857 and $545 \mathrm{GHz}$. Temperature maps were derived at the common resolution of the 3 bands used $\left(5^{\prime}\right)$ and at lower resolution for further analysis. In each case, the emission was computed in the photometric channels of the instruments used (IRAS, Planck, and WMAP), including the colour corrections, using the actual transmission profiles for each instrument, and following the flux convention description given in the respective explanatory supplements.

In order to derive the thermal dust temperature, we use the same strategy as described in Planck Collaboration (2011o). To 
Table 3. Dust temperature, $\beta$ values and fit reduced $\chi^{2}$ for various methods experimented to derive the temperature maps.

\begin{tabular}{llll}
\hline \hline Method & $\begin{array}{l}T_{\mathrm{D}} \pm \Delta T_{\mathrm{D}} \\
{[\mathrm{K}]}\end{array}$ & $\beta \pm \Delta \beta$ & $\chi^{2}$ \\
\hline LMC: & & & \\
free $\beta$ & $21.0 \pm 1.9$ & $1.48 \pm 0.25$ & 1.91 \\
fixed $\beta$ & $20.7 \pm 1.7$ & $1.5^{\dagger}$ & 1.73 \\
fixed $\beta$ & $19.2 \pm 1.6$ & 1.8 & 1.63 \\
fixed $\beta$ & $18.3 \pm 1.6$ & 2.0 & 1.57 \\
dustem & $17.7 \pm 1.6$ & - & 1.60 \\
\hline SMC: & & & \\
free $\beta$ & $22.3 \pm 2.3$ & $1.21 \pm 0.27$ & 2.28 \\
fixed $\beta$ & $21.6 \pm 1.9$ & $1.2^{\dagger}$ & 1.90 \\
fixed $\beta$ & $18.8 \pm 1.7$ & 1.8 & 9.94 \\
fixed $\beta$ & $17.9 \pm 1.6$ & 2.0 & 14.0 \\
dustem & $17.3 \pm 1.6$ & - & 12.66 \\
\hline
\end{tabular}

Notes. The values listed are median values in the SED integration region for each galaxy. ${ }^{(\dagger)}$ Fixed $\beta$ model used in this paper.

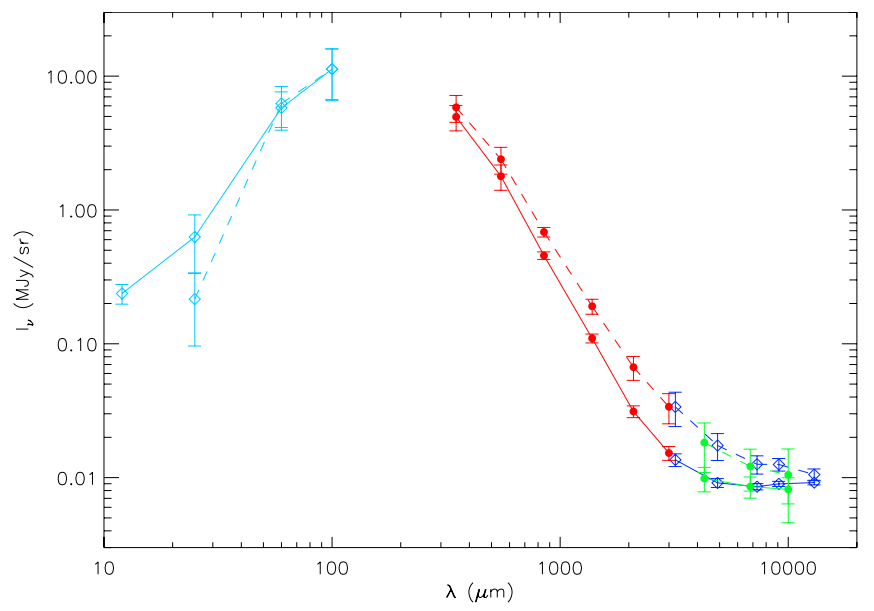

Fig. 6. Integrated SEDs of the LMC (solid) and SMC (dashed) after $\mathrm{CMB}$ and galactic foreground subtraction, including data from PlanckHFI (red), Planck-LFI (green), IRAS-IRIS (light blue) and WMAP (dark blue). The uncertainties shown are $\pm 3 \sigma$. The SMC SED was scaled by a factor 4 providing normalization at $100 \mu \mathrm{m}$.

minimise computation time, the predictions of the model were tabulated for a large set of parameters $\left(T_{\mathrm{D}}, \beta\right)$. For each map pixel, the $\chi^{2}$ was computed for each entry of the table and the shape of the $\chi^{2}$ distribution around the minimum value was used to derive the uncertainty on the free parameters. This includes the effect of the data variance $\sigma_{\text {II }}^{2}$ and the absolute uncertainties.

We explored several options for deriving maps of the apparent dust temperature, which are summarised in Table 3. We first fitted each pixel of the maps with a modified black body of the form $I_{v} \propto v^{\beta} B_{v}\left(T_{\mathrm{D}}\right)$ in the above spectral range (method referred to as "free $\beta$ " in Table 3). The median values of $T_{\mathrm{D}}$ and $\beta$ derived using this method are given in the first line of Table 3 . This led to median $\beta$ values of $\beta_{\mathrm{LMC}} \simeq 1.5$ and $\beta_{\mathrm{SMC}} \simeq 1.2$ for the LMC and SMC respectively. Note that the value for the LMC is consistent with that derived using a combination of the IRAS and Herschel data by Gordon et al. (2010). However, inspection of the corresponding maps show correlated variations of the two parameters which were also correlated with the noise level in the maps. This suggests that the spurious values probably originated from the correlation in parameter space and the presence of noise in the data, particularly in low brightness regions of the maps.
We then performed fits of the FIR emission using the fixed $\beta$ values, with the $\beta$ values given above for the two galaxies (method referred to as "fixed $\beta$ " and marked with a ${ }^{\dagger}$ in Table 3 ). Although the median reduced $\chi^{2}$ was slightly higher than for the "free $\beta$ " method, the temperature maps showed fewer spurious values, in particular in low brightness regions. This resulted in a more coherent distribution of the temperature values than in the "free $\beta$ " case. Since we later used the temperature maps to investigate the spectral distribution of the dust optical depth, and the dust temperature is a source of uncertainty, we adopted the "fixed $\beta$ " maps in what follows. The corresponding temperature maps for the LMC and SMC are shown in Fig. 7.

The calculations were also carried out for $\beta=1.8$, which is the average Galactic value (Planck Collaboration 2011o,t), in order to be able to derive dust emissivity values under the same assumption as in the MW. We also experimented using the "standard" $\beta=2$ value.

\subsection{Angular distribution of dust temperature}

\subsubsection{LMC}

The temperature map derived here for the LMC shows a similar distribution to the one derived from IRAS and Spitzer data by Bernard et al. (2008). The highest temperatures are observed toward 30-Dor and are of the order of $24 \mathrm{~K}$. The large scale distribution shows the existence of an inner warm arm, which follows the distribution of massive star formation as traced by known $\mathrm{H}_{\text {II }}$ regions. Figure 8 shows that the warm dust at $T_{\mathrm{D}}>$ $20 \mathrm{~K}$ in the LMC is reasonably well correlated with $\mathrm{H}_{\alpha}$ emission, indicating that it is heated by the increased radiation field induced by massive star formation. Two regions departing from the correlation are visible to the SW of the 30-Dor at $\alpha_{2000}=$ $05^{\mathrm{h}} 40^{\mathrm{m}}, \delta_{2000}=-70^{\circ} 30^{\prime}$ and $\alpha_{2000}=05^{\mathrm{h}} 40^{\mathrm{m}}, \delta_{2000}=-72^{\circ} 30^{\prime}$. The first region was already identified in Bernard et al. (2008) who showed that this low column density region was unexpectedly warm given that no star formation is taking place in this area. They proposed that this could be an artefact of the IRASIRIS $100 \mu \mathrm{m}$ used in the analysis, possibly due to IRAS gain variations across the bright 30 -Dor source. However, the analysis carried out here and the comparison between temperature maps obtained with and without the MW background subtraction suggests that the temperature measurement has been affected by the low surface brightness. This emphasises the necessity of correcting for temperature bias at low brightness. The second region is close to the edge of the map and is probably also affected by low surface brightness.

The Planck data however reveals cold areas in the outer regions of the LMC, which were not seen using the Spitzer data. This is essentially due to the large coverage of the Planck data with respect to the limited region which was covered by the Spitzer data. The south of the LMC exhibits a string of cold regions with $T_{\mathrm{D}}<20 \mathrm{~K}$. These regions correspond without exception to known molecular clouds when they fall in the region covered by the CO survey. They also correspond to peaks of the dust optical depth as derived in the following sections. Similarly, a set of cold regions exist at the northwest periphery of the LMC. The comparison with the distribution of CO clouds is shown in Fig. 8. As already noticed in Bernard et al. (2008), there is no systematic correlation between cold dust and the presence of molecular material, at least in the inner regions of the LMC. This was confirmed by the statistical characteristics of the IR properties of LMC molecular clouds established by Paradis et al. (2010), which showed no systematic trend for molecular regions to be 

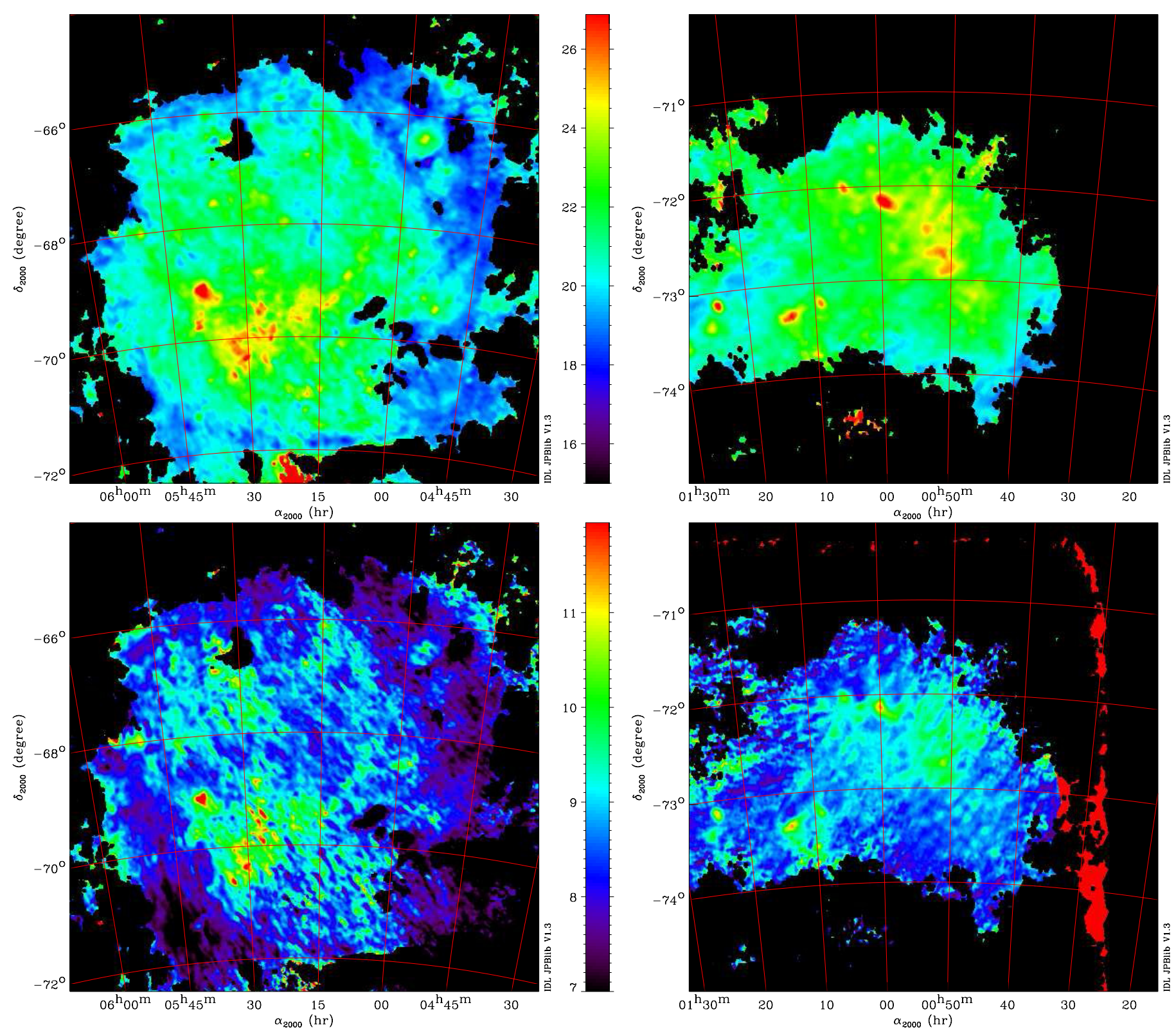

Fig. 7. Upper panels: dust temperature maps for the LMC (left) and SMC (right) computed from the foreground subtracted maps using the IRAS-IRIS $100 \mu \mathrm{m}$, HFI 857 and $545 \mathrm{GHz}$ maps, using a fixed $\beta_{\mathrm{LMC}}=1.5$ and $\beta_{\mathrm{SMC}}=1.2$. Lower panels: relative uncertainties on the dust temperature at the same resolution, expressed as percentages.

colder than their surrounding neutral material. However, toward the outer regions of the LMC, molecular clouds systematically appear to show a decrease in the dust temperature. This difference may be due to the absence of star formation activity in the outer regions and/or to less mixing along the line of sight.

Gordon et al. (2010) derived a dust temperature map from the Herschel data (HERITAGE program) for a fraction of the LMC observed during the science demonstration phase (SDP). They used the $100 \mu \mathrm{m}$ to $350 \mu \mathrm{m}$ IRAS and Herschel bands to constrain the temperature. They found that the temperatures derived this way only differ from those derived by Bernard et al. (2008) by up to $10 \%$. The SDP field covered an elongated region across the LMC roughly oriented north-south. This strip crossed the warm inner arm, which is also clearly seen in their map. However, we stress that, in their study, a gradient was removed along the strip based on the values at the edges. The fact that we detect significantly colder than average dust in the outer cold arm of the LMC underlines the need to take those variations into account when subtracting background emission in the Herschel data.

\subsubsection{SMC}

The temperature map derived for the SMC shows more moderate temperature variations than those observed in the LMC but peaks corresponding to well known HII regions are clearly identified, and the overall dust temperature correlates with $\mathrm{H}_{\alpha}$ emission, as shown in Fig. 9. In particular, the massive star forming region SMC-N66 (Henize 1956) $\left(\alpha_{2000}=\right.$ $\left.00^{\mathrm{h}} 59^{\mathrm{m}} 27.40^{\mathrm{s}}, \delta_{2000}=-72^{\circ} 10^{\prime} 11^{\prime \prime}\right)$ corresponds to the highest temperature $(\sim 20 \mathrm{~K})$ in the SMC. Other well known star forming regions like SMC-N83/84 $\left(01^{\mathrm{h}} 14^{\mathrm{m}} 21.0^{\mathrm{s}},-73^{\circ} 17^{\prime} 12^{\prime \prime}\right)$, N $81\left(01^{\mathrm{h}} 09^{\mathrm{m}} 13.6^{\mathrm{s}},-73^{\circ} 11^{\prime} 41^{\prime \prime}\right)$, N88/89/90 (in the wing: $01^{\mathrm{h}} 24^{\mathrm{m}} 08.1^{\mathrm{s}},-73^{\circ} 08^{\prime} 55^{\prime \prime}$ ) and DEM S54 (at the centre of 

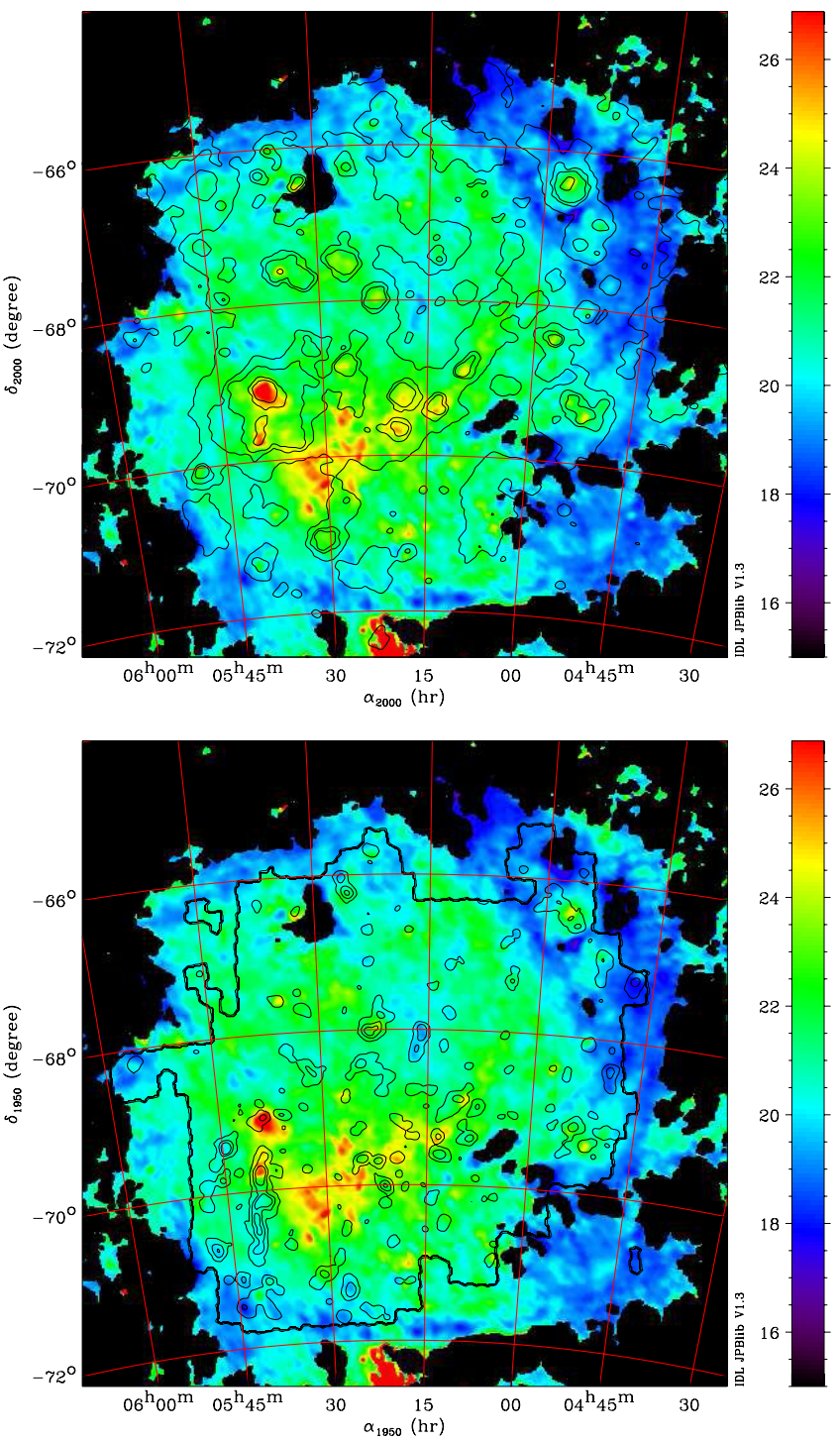

Fig. 8. Comparison between the dust temperature map of the LMC with $\mathrm{H}_{\alpha}$ (top) and $\mathrm{CO}$ emission (bottom). The $\mathrm{CO}$ contours are at 0.5, 2, 4 and $10 \mathrm{~K} \mathrm{~km} \mathrm{~s}^{-1} . \mathrm{H}_{\alpha}$ contours are at 1, 10, 50, 100, 500 and 1000 Rayleigh. The thick line shows the edge of the available CO surveys.

the main bar: $\left.00^{\mathrm{h}} 50^{\mathrm{m}} 25.9^{\mathrm{s}},-72^{\circ} 53^{\prime} 10^{\prime \prime}\right)$ appear as temperature peaks compared to the surroundings. In contrast, the infraredbright star forming region $\mathrm{N} 76$ (located at the northeast of $\mathrm{N} 66$ : $\left.01^{\mathrm{h}} 04^{\mathrm{m}} 01.2^{\mathrm{s}},-72^{\circ} 01^{\prime} 52^{\prime \prime}\right)$ and southwest star-forming complex do not stand out. This could be due to the presence of an extended warm component that we observe in the main bar and that seems spatially related to the diffuse $\mathrm{H}_{\alpha}$ emission.

\subsection{Optical depth determination}

The optical depth is derived from:

$$
\tau(v)=\frac{I_{v}}{B_{v}\left(T_{\mathrm{d}}\right)},
$$

where $B_{v}$ is the Planck function. We used resolution-matched maps of $T_{\mathrm{D}}$ and $I_{v}$ and derived $\tau$ maps at the various resolutions of the data used here. The uncertainty on $\tau(\Delta \tau)$ is computed as:

$\Delta \tau(v)=\tau\left(\frac{\sigma_{\mathrm{II}}^{2}}{I_{v}^{2}}+\left(\frac{\delta B_{v}\left(T_{\mathrm{D}}\right)}{\delta T} \frac{\Delta T_{\mathrm{D}}}{B_{v}\left(T_{\mathrm{D}}\right)}\right)^{2}\right)^{1 / 2}$.
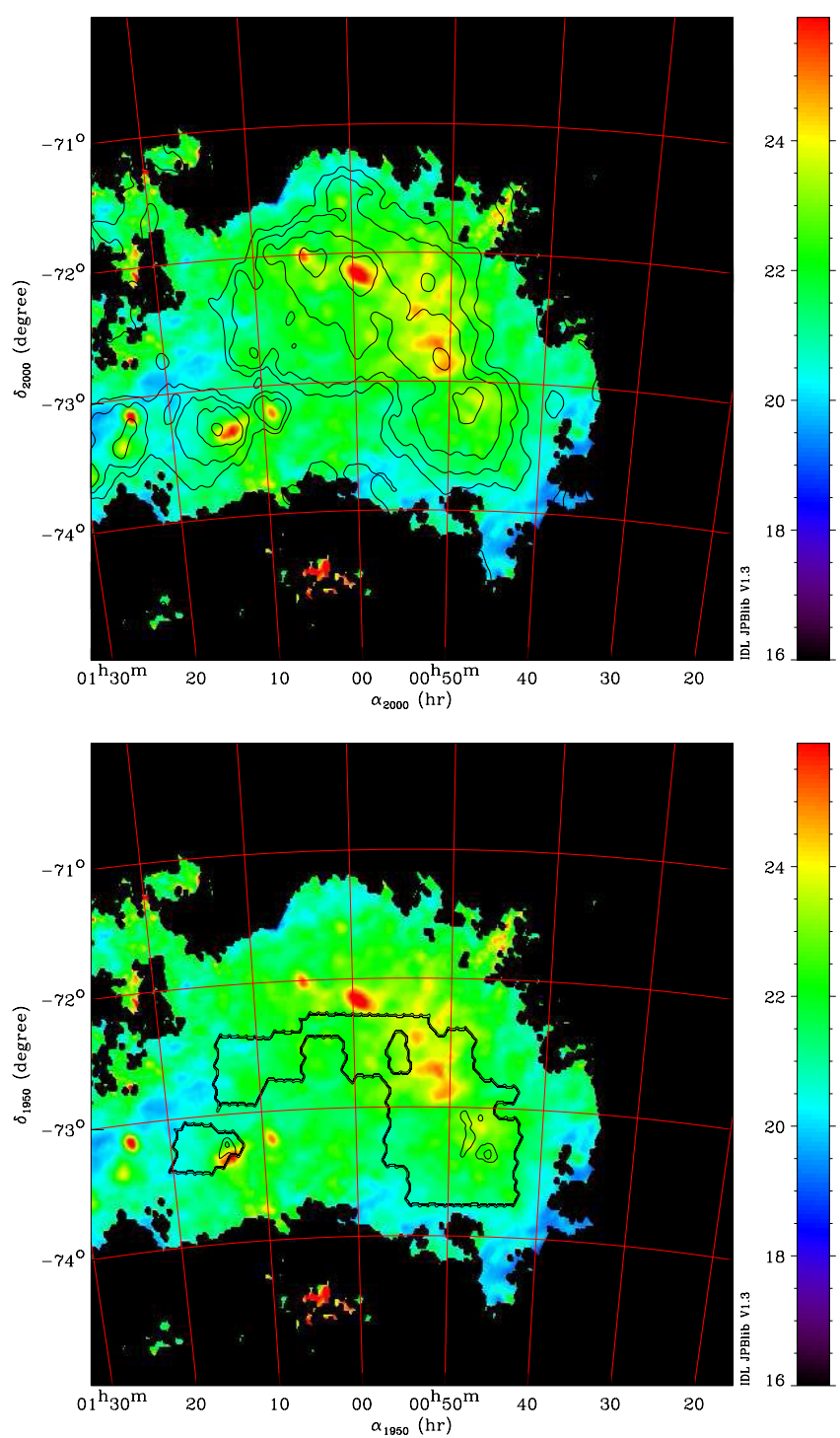

Fig. 9. Comparison between the dust temperature map of the SMC with $\mathrm{H}_{\alpha}$ (top) and $\mathrm{CO}$ emission (bottom). The $\mathrm{CO}$ contours are at $0.5,1$ and $1.5 \mathrm{~K} \mathrm{~km} \mathrm{~s}^{-1} \cdot \mathrm{H}_{\alpha}$ contours are at 1, 5, 30 and 100 Rayleigh. The thick line shows the edge of the available CO surveys.

The optical depth and optical depth uncertainty maps derived at $857 \mathrm{GHz}$ are shown in Figs. 10 and 11 for the LMC and the SMC respectively.

\section{Discussion}

\subsection{The millimetre excess}

Bot et al. (2010b) (see Fig. 5) found that the integrated SED of the LMC and more noticeably of the SMC showed excess millimetre emission with respect to a dust and free-free/synchrotron model based respectively on the FIR and the radio data. They investigated several causes for this excess. They noticed that the excess had precisely the colours of CMB fluctuations. They performed simulations by placing the LMC and SMC structure at various positions over a CMB simulated sky, and concluded that a CMB origin was unlikely, but not excluded. Other proposed origins for the excess included the presence of very cold dust, spinning dust or large modifications of the optical properties of thermal dust. 

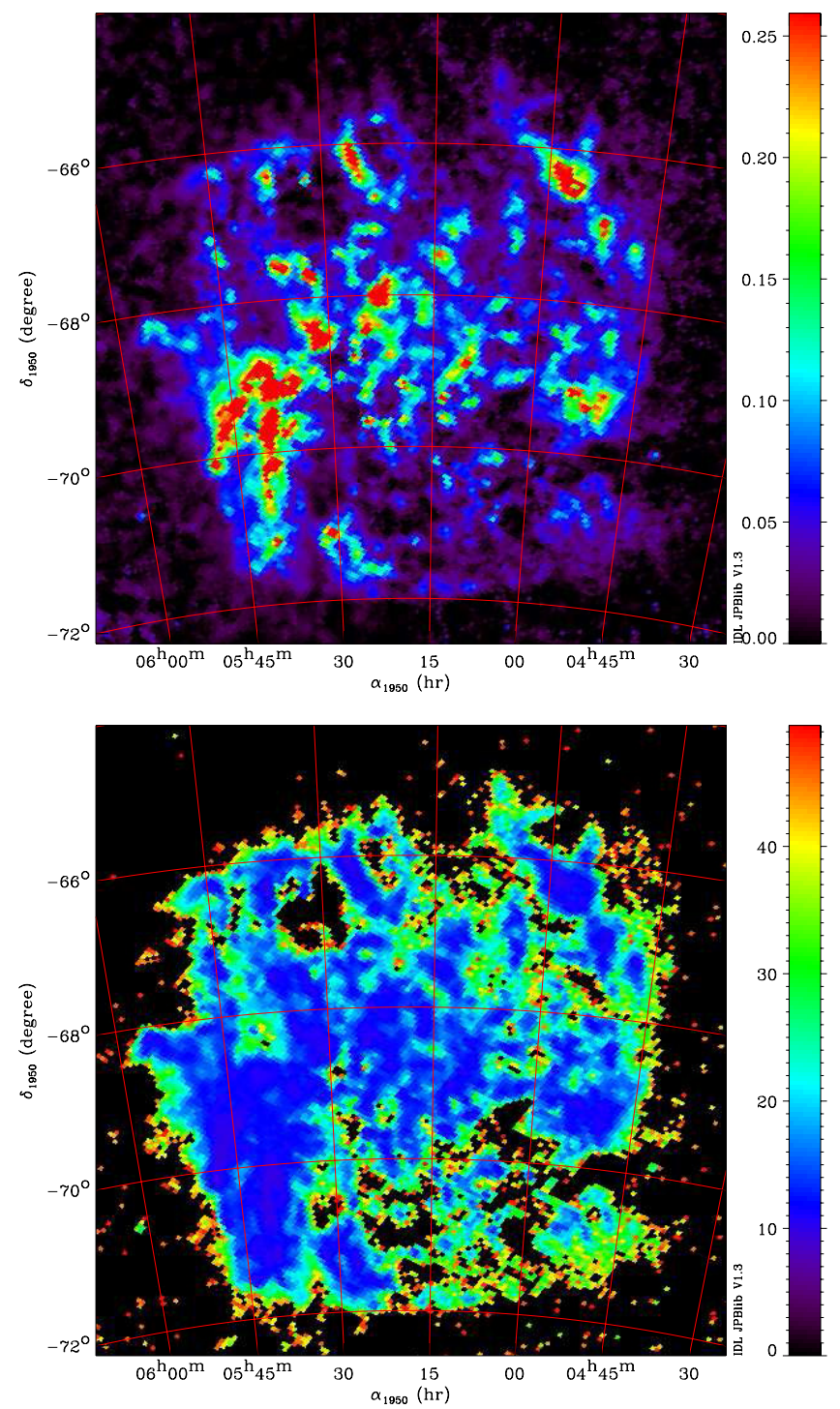

Fig. 10. Upper panel: map of the dust optical depths of the LMC at HFI $217 \mathrm{GHz}$. Units are $10^{4} \times \tau$. Lower panel: map of the dust optical depth relative uncertainty of the LMC at HFI $217 \mathrm{GHz}$ in percent. Black pixels in the maps are masked and have relative uncertainties larger than $50 \%$.

Very cold dust $\left(T_{\mathrm{D}} \simeq 5-7 \mathrm{~K}\right)$ has been advocated to explain the flattening of the millimetre emission observed in more distant low metallicity galaxies (e.g. Galliano et al. 2005). However, this has usually led to very large masses, and the existence of such very cold dust remains controversial and difficult to understand in low metallicity systems where the stronger star formation rate and the lower dust abundances prevent efficient screening from UV photons.

Bot et al. (2010b) applied spinning dust models to fit the SMC and LMC SEDs and found a plausible match. However, since the observed excess peaked at a significantly higher frequency than observed for spinning dust in other regions (e.g. Planck Collaboration 2011p), their fit required extreme density and excitation conditions for the small dust particles.

Using the two level system (TLS) model by Meny et al. (2007) for the long wavelength emission of amorphous solids also proved plausible for the LMC, but a convincing fit could not be found for the SMC, essentially because the model could not reproduce the shape of the excess.
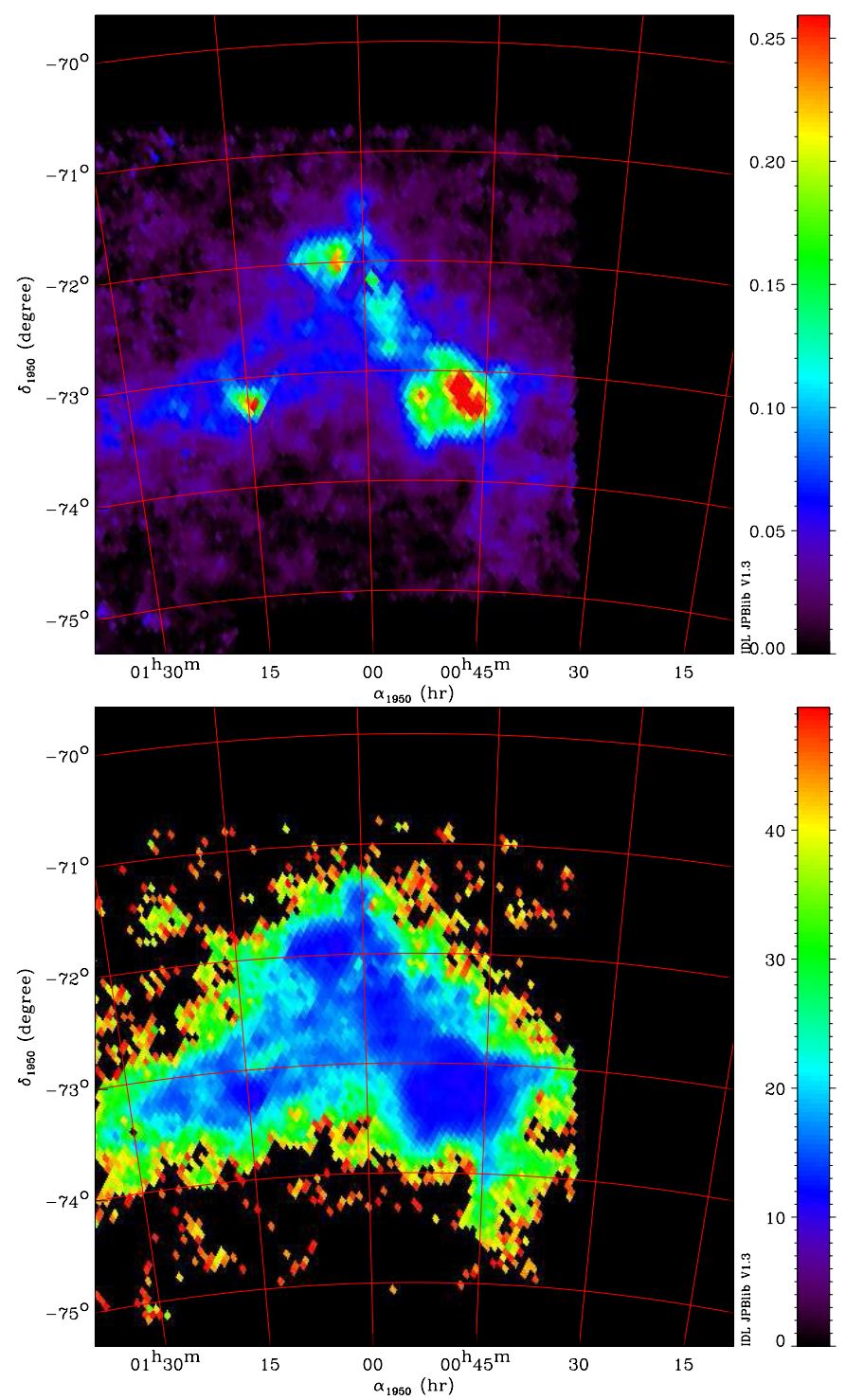

Fig. 11. Upper panel: map of the dust optical depths of the SMC at HFI $217 \mathrm{GHz}$. Units are $10^{4} \times \tau$. Lower panel: map of the dust optical depth relative uncertainty at HFI $217 \mathrm{GHz}$ in percent. Black pixels in the maps are masked and have relative uncertainties larger than 50\%.

The study carried out here, which takes advantage of the Planck measurements to constrain the CMB foreground fluctuations towards the two galaxies, shows that part of the excess observed toward the SMC cannot be accounted for by the fluctuations of the background CMB, as discussed in Sect. 2.3.2, but the intensity of the excess has been greatly reduced compared to that found by Bot et al. (2010b).

To give more insight into this excess, we built a map to trace the spatial distribution of the excess emission in the SMC. To do this, we applied the fitting procedure performed for the integrated SEDs of the Magellanic Cloud, to each point of the SMC at the angular resolution of the LFI lowest frequency channel. The SEDs were build at each point using the CMB and foreground-subtracted data. To model the SEDs, we assumed that, at each point, the SEDs are dominated by free-free and dust emission up to the longest wavelengths covered by Planck, following what is observed for the integrated emission of the SMC (see Fig. 5). The free-free component at each wavelength was extrapolated from the $\mathrm{H}_{\alpha}$ emission (as in Sect. 3), assuming that 


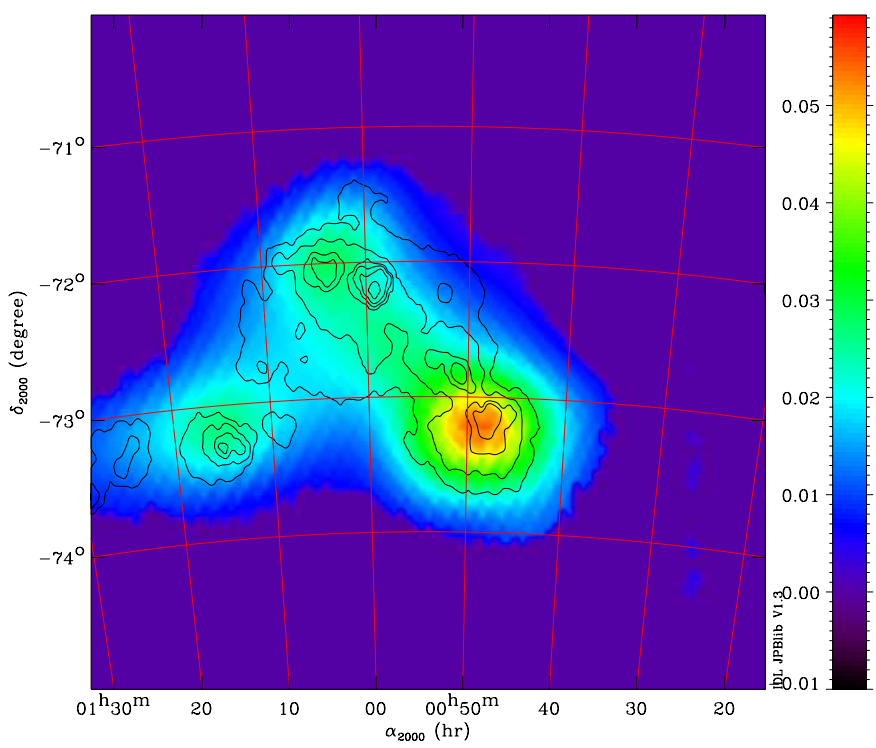

Fig. 12. Map of the millimetre excess in the $\mathrm{SMC}$ at $3 \mathrm{~mm}$ computed at the resolution of the LFI-28.5 GHz channel, after free-free subtraction. The contours show the $\mathrm{H}_{\alpha}$ distribution.

the extinction in the SMC is negligible. The thermal dust emission was then fitted to the data points at $\lambda_{\text {ref }}<550 \mu \mathrm{m}$, using the Draine et al. (2007) dust model. The millimetre excess was then defined as the difference between the data and the dust-andfree-free model. The resulting spatial distribution of the excess emission at $100 \mathrm{GHz}(3 \mathrm{~mm})$ is shown in Fig. 12. This shows that the peak of the excess is located at the southwest tip of the bar. This region also corresponds to the maximum of the optical depth derived from the FIR and shown in Fig. 11, and the overall excess spatial distribution is consistent with being proportional to the dust column density.

\subsection{FIR dust emissivity}

The wavelength dependence of the average dust optical depth in the LMC and SMC is shown in Fig. 13. The spectral index of the emissivity in the FIR is consistent with $\beta=1.5$ and $\beta=1.2$ for the LMC and the SMC respectively. This value for the LMC is consistent with findings by Gordon et al. (2010) using the Herschel data. We see no hint of a change of the spectral index with wavelength for the LMC. In contrast, the SMC SED clearly flattens at $\lambda>800 \mu \mathrm{m}$ to reach extremely flat $\beta$ values around $\lambda=3 \mathrm{~mm}$. The dust emissivities as interpolated using the power laws shown in Fig. 13 can be compared to the reference value for the solar neighbourhood of $\tau / N_{\mathrm{H}}=10^{-25} \mathrm{~cm}^{2}$ at $250 \mu \mathrm{m}$ (Boulanger et al. 1996). This comparison indicates lower than solar dust abundances for the two galaxies, by about $1 / 2.4$ and $1 / 13$ respectively. This is in rough agreement with the metallicity for the LMC and significantly lower than the metallicity for the SMC.

Gordon et al. (2010) found hints of such excess emission around $500 \mu \mathrm{m}$ in the LMC Herschel data, but this remained within the current calibration uncertainties of the Herschel Spectral and Photometric Imaging REceiver (SPIRE) instrument. We also observe that the $500 \mu$ m optical depth of the LMC is slightly higher (by 8.4\%) than the power law extrapolation shown in Fig. 13, but this is only marginally larger than the $1 \sigma$ uncertainty at this wavelength. In any case, data points at longer

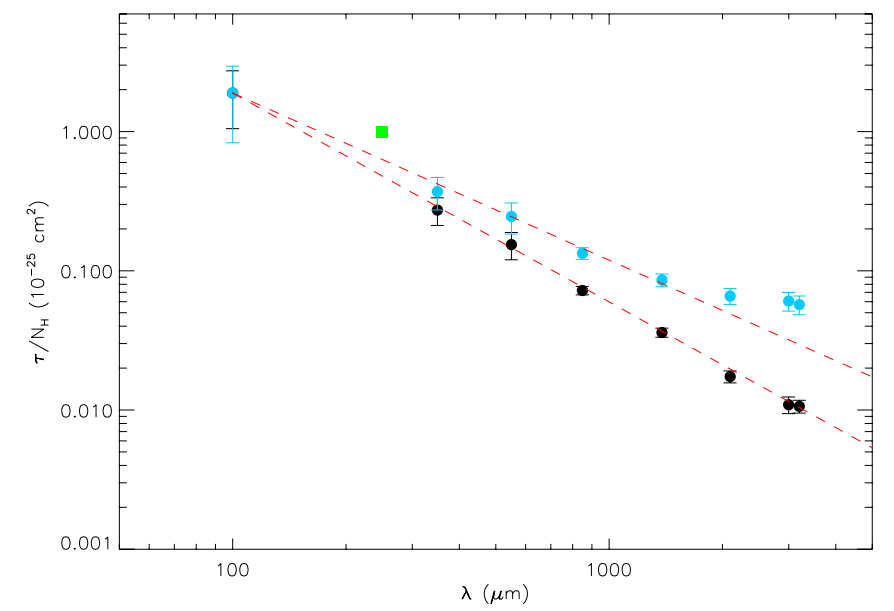

Fig. 13. Average dust optical depth of the LMC (black) and SMC (blue) obtained from the CMB, foreground and free-free subtracted SEDs. The average is taken in the same regions as Fig. 5. The SMC SED was normalised (multiplied by 6.31) to that of the LMC at $100 \mu \mathrm{m}$. The square symbol (green) shows the reference value for the solar neighbourhood by Boulanger et al. (1996). The dashed lines show $\tau \propto v^{1.5}$ and $\tau \propto v^{1.2}$ normalised at $100 \mu \mathrm{m}$. Error bars are $\pm 3-\sigma$.

wavelengths do not support the existence of excess emission in addition to a $\beta=1.5$ power law for the LMC.

\subsection{Possible interpretation of the excess}

In this section, we attempt to fit the SED of the SMC using various models which have been proposed to explain excess submm emission, in addition to a single dust emissivity power-law for thermal dust. Figure 14 shows several fits to the FIR-Submm SED of the SMC.

The first fit uses the Finkbeiner et al. (1999) model $\sharp 7$ (see their Table 3), which was designed to explain the flatterthan-expected emission spectrum of our Galaxy as observed by FIRAS. Here we use this model to assess the possibility that the millimetre excess is due to very cold dust. The model was fitted using the same $\beta$ values as in Finkbeiner et al. (1999) for the two dust components $\left(\beta_{\text {warm }}=2.6, \beta_{\text {cold }}=1.5\right)$. It also assumes the same type of relationship between the cold and warm dust temperatures, which in the model reflects the fact that both dust species are subjected to the same radiation field. However, we allow the IR/optical opacity ratio $\left(q_{\text {cold }} / q_{\text {warm }}\right)$ to vary, so that the ratio between the warm and cold dust temperatures is free to vary. We also leave the cold dust component abundance $\left(f_{\text {cold }}\right)$ and the warm dust temperature $\left(T_{\text {warm }}\right)$ as free parameters. The best fit values are found for $T_{\text {warm }}=16.4 \mathrm{~K}$ $q_{\text {cold }} / q_{\text {warm }}=168.2, f_{\text {cold }}=8.7 \times 10^{-3}$. For these parameters, the temperature of the cold component is $T_{\text {cold }}=5.9 \mathrm{~K}$. This cold temperature is needed to reproduce part of the millimetre excess but is much colder than that obtained by Finkbeiner et al. (1999) for the Galaxy $\left(T_{\text {cold }}=9.6 \mathrm{~K}\right)$. It is obtained at the expense of strongly increasing the IR/optical opacity ratio for the cold component $\left(q_{\text {cold }} / q_{\text {warm }}\right.$ increased by a factor 15$)$, which controls the $T_{\text {cold }} / T_{\text {warm }}$ ratio. The mass fraction of the cold component derived here, $f_{\text {cold }}$, is about 4 times lower than that derived for the MW in Finkbeiner et al. (1999), which compensates for the increased $q_{\text {cold }} / q_{\text {warm. }}$. It is apparent that, despite invoking a much larger IR/optical opacity ratio for the cold particles, such a model has difficulties producing the submm excess above about $\lambda=2 \mathrm{~mm}$. 

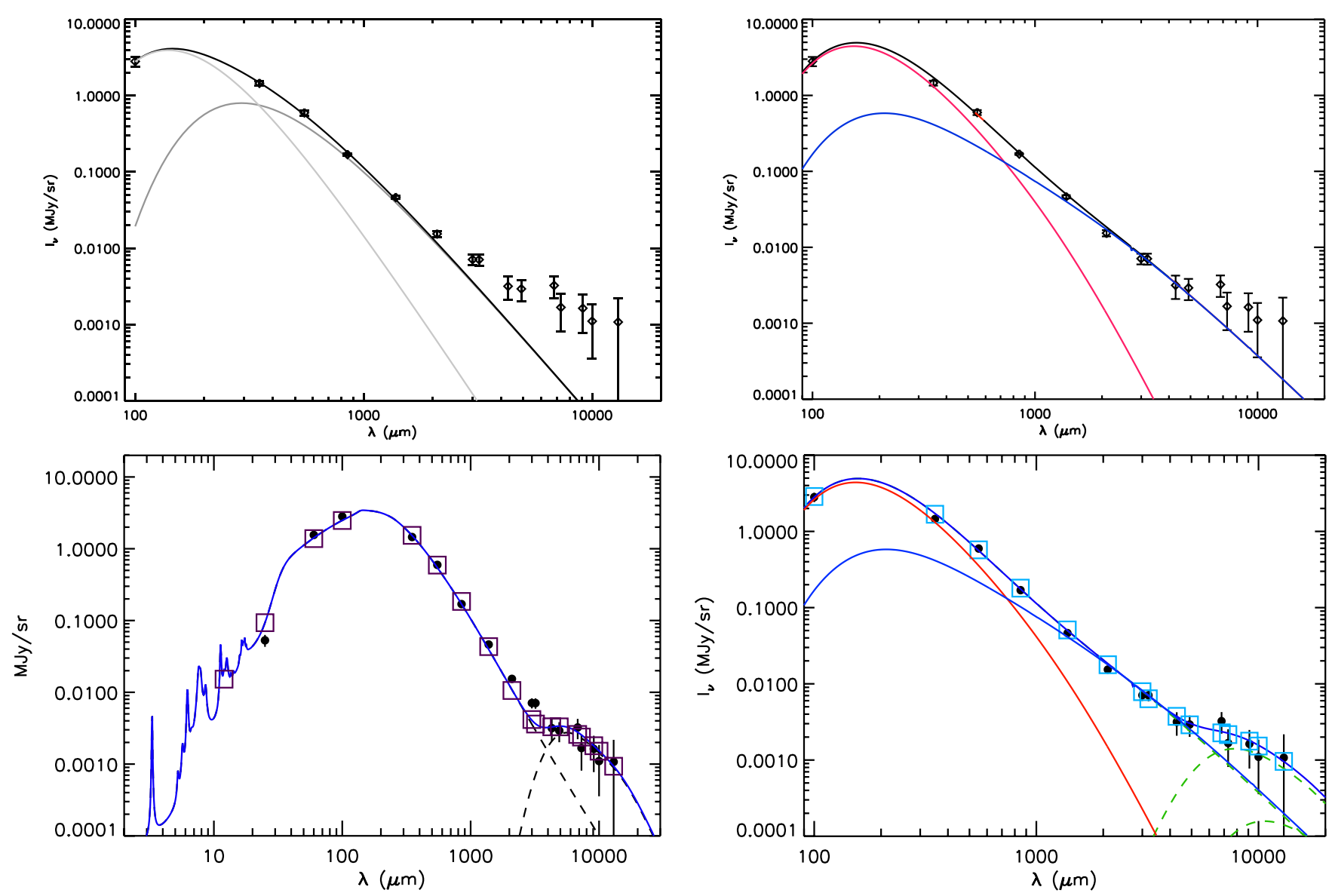

Fig. 14. Fit of the SMC SED using the Finkbeiner model (upper left), the TLS model (upper right), the spinning dust model (lower left) and a combination of TLS and spinning dust models (lower right). In the lower panels, the squares represent the flux in each band predicted by the best model (the blue line).

The second fit employs the TLS model developed by Meny et al. (2007). The fit was obtained by minimizing $\chi^{2}$ in the range $100 \mu \mathrm{m}<\lambda<5 \mathrm{~mm}$ against the following 3 parameters: $T_{\mathrm{D}}$ the dust temperature, $l_{\text {cor }}$ the correlation length of defects in the material and A the density of TLS sites in the material composing the grains. The values derived for these parameters are $T_{\mathrm{D}}=18.9 \mathrm{~K}, l_{\text {cor }}=12.85 \mathrm{~nm}$ and $A=7.678$. The reduced $\chi^{2}$ for these parameters is $\chi^{2}=2.56$. Compared to the best values found from Paradis (2007) for the MW $\left(T_{\mathrm{D}}=17.9 \mathrm{~K}\right.$, $l_{\text {cor }}=12.85 \mathrm{~nm}, A=2.42$ ), this indicates dust material with more TLS sites (more mechanical defects) but a similar defect correlation length compared with the dust dominating the MW emission. Note that a grey-body fit of the same SED over the same frequency range leads to $T_{\mathrm{D}}=18.9 \mathrm{~K}$ and $\beta=0.74$ and a reduced $\chi^{2}=6.12$, showing that the TLS model reproduces the data better than a single grey-body fit.

The third fit uses spinning dust. In this case, the thermal dust emission was reproduced using the Draine \& Li (2007) dust model. The remaining excess was then fitted with the spinning dust model described in Silsbee et al. (2011). Spinning dust emission has been proved to be sensitive to the neutral and ionised gas densities and to the size distribution of dust grains. The radiation field and the size distributions were taken to be the same as for the Draine \& Li (2007) model. The relevant gas parameters were computed with CLOUDY (Ferland et al. 1998): we took the parameters from the optically thin zone of isochoric simulations. In both cases, we took into account the lower metallicity and dust grains abundance with respect to the MW. The electric dipole moment distribution of grains was taken as in Draine \& Lazarian (1998b) ${ }^{2}$. The fit was obtained by adding two components according to the PDR fraction inferred from the thermal emission model: a diffuse medium with $n_{\mathrm{H}}=30 \mathrm{~cm}^{-3}$ and $100 \%$ of the PAH mass expected from the IR modelling, and a denser medium with $n_{\mathrm{H}}=5000 \mathrm{~cm}^{-3}$ and $82 \%$ of the expected PAH mass.

The fourth fit is a combination of the TLS model and the spinning dust model presented above.

It is clear from Fig. 14 that the model combining TLS and spinning dust is the only one of the proposed models giving a satisfactory fit to the millimetre excess observed in the SMC over the whole spectral range. In addition, it alleviates the need for using more PAH than allowed by the NIR emission to produce the required level of spinning dust emission observed. Alternative models, however, cannot be excluded. For instance, very large grains $(a>30 \mu \mathrm{m})$ that would be efficient radiators (Rowan-Robinson 1992) could create emission in the millimetre wavelengths without too much mass. Exploring this possibility would require specific modelling. This is beyond the scope of the current article but should be explored in future studies.

\footnotetext{
${ }^{2}$ Ysard \& Verstraete (2010) showed that it is in good agreement with the anomalous emission extracted from the WMAP data.
} 


\section{Conclusions}

We assessed the existence and investigated the origin of millimetre excess emission in the LMC and the SMC using the Planck data. In the framework of this preliminary analysis, we focused our interest on the thermal dust emission from the galaxies, which we isolated by subtracting other unrelated components such as galactic foreground, CMB fluctuations and freefree emission. More detailed studies in the future will involve full multi-component analysis or the use of more sophisticated component separation algorithms exploiting all spatial and spectral information available in the data.

The integrated SED of the two galaxies before subtraction of the foreground (Milky Way) and background (CMB fluctuations) emission are in good agreement with previous determinations.

The background CMB contribution was first subtracted using an ILC method performed locally around the two galaxies. The uncertainty of this contribution was measured through a detailed Monte-Carlo simulation. We subtracted the foreground emission from the Milky Way using a Galactic Hi template and the proper dust emissivity derived in a region surrounding the two galaxies and dominated by MW emission. We also subtracted the free-free contribution from ionised gas in the galaxies, using the $\mathrm{H}_{\alpha}$ emission, taking advantage of the low extinction in those galaxies. The remaining emission of both galaxies correlates with the gas emission of the LMC and SMC.

We showed that the excess previously reported in the LMC can be fully explained by CMB fluctuations. For the SMC, subtracting the CMB fluctuations decreases the intensity of the excess but a significant millimetre emission above the expected thermal dust, free-free and synchrotron emission remains.

We combined the Planck and IRAS-IRIS data at $100 \mu \mathrm{m}$ to produce thermal dust temperature and optical depth maps of the two galaxies. The LMC temperature map shows the presence of a warm inner arm already found with the Spitzer data, but also shows the existence of a previously unidentified cold outer arm. Several cold regions were found along this arm, some of which are associated to known molecular clouds.

We used the dust optical depth maps to constrain the thermal dust emissivity spectral index $(\beta)$. The average spectral index in the FIR $(\lambda<500 \mu \mathrm{m})$ is found to be consistent with $\beta=1.5$ and $\beta=1.2$ for the LMC and the SMC respectively. This is significantly flatter than is observed in the Milky Way. The absolute values of the emissivities in the FIR, when compared to that in our solar neighbourhood, are compatible with $\mathrm{D} / \mathrm{G}$ mass ratios of $1 / 2.4$ and $1 / 13$ for the LMC and SMC. This is compatible with the metallicity of the LMC but significantly lower than the metallicity of the SMC. In the submm, the LMC SED remains consistent with $\beta=1.5$, while the SED of the SMC is even flatter.

The spatial distribution of the mm excess in the SMC appears to follow the general pattern of the gas distribution. It therefore appears unlikely that the excess could originate from very cold dust. Indeed, this is confirmed by attempts to fit the SMC emission SED with models with two dust components, which led to poor fits. Alternative models, such as emission excess due to the amorphous nature of large grains, are likely to provide a natural explanation for the observed SEDs, although spinning dust is needed to explain the SED above $\lambda=3 \mathrm{~mm}$.

Acknowledgements. A description of the Planck Collaboration and a list of its members can be found at http://www.rssd.esa.int/index.php? project=PLANCK\&page=Planck_Collaboration

\section{References}

Anderson, N., \& Watson, W. D. 1993, A\&A, 270, 477

Bendo, G. J., Dale, D. A., Draine, B. T., et al. 2006, ApJ, 652, 283

Bennett, C. L., Halpern, M., Hinshaw, G., et al. 2003, ApJS, 148, 1

Bernard, J., Reach, W. T., Paradis, D., et al. 2008, AJ, 136, 919

Bolatto, A. D., Simon, J. D., Stanimirović, S., et al. 2007, ApJ, 655, 212

Bot, C., Boulanger, F., Lagache, G., Cambrésy, L., \& Egret, D. 2004, A\&A, 423, 567

Bot, C., Rubio, M., Boulanger, F., et al. 2010a, A\&A, 524, A52

Bot, C., Ysard, N., Paradis, D., et al. 2010b, A\&A, 523, A20

Boulanger, F., Abergel, A., Bernard, J., et al. 1996, A\&A, 312, 256

Dobashi, K., Bernard, J., Hughes, A., et al. 2008, A\&A, 484, 205

Draine, B. T., \& Lazarian, A. 1998a, ApJ, 494, L19

Draine, B. T., \& Lazarian, A. 1998b, ApJ, 508, 157

Draine, B. T., \& Li, A. 2007, ApJ, 657, 810

Draine, B. T., Dale, D. A., Bendo, G., et al. 2007, ApJ, 663, 866

Dumke, M., Krause, M., \& Wielebinski, R. 2003, in Rev. Mex. Astron. Astrof. Conf. Ser., ed. V. Avila-Reese, C. Firmani, C. S. Frenk, \& C. Allen, 27, 17, 185

Dupac, X., Bernard, J., Boudet, N., et al. 2003, A\&A, 404, L11

Eriksen, H. K., Banday, A. J., Górski, K. M., \& Lilje, P. B. 2004, ApJ, 612, 633 Ferland, G. J., Korista, K. T., Verner, D. A., et al. 1998, PASP, 110, 761

Ferrara, A., \& Dettmar, R. 1994, ApJ, 427, 155

Finkbeiner, D. P., Davis, M., \& Schlegel, D. J. 1999, ApJ, 524, 867

Fukui, Y., Mizuno, N., Yamaguchi, R., et al. 1999, PASJ, 51, 745

Fukui, Y., Kawamura, A., Minamidani, T., et al. 2008, ApJS, 178, 56

Galametz, M., Madden, S., Galliano, F., et al. 2009, A\&A, 508, 645

Galametz, M., Madden, S. C., Galliano, F., et al. 2010, A\&A, 518, L55

Galliano, F., Madden, S. C., Jones, A. P., et al. 2003, A\&A, 407, 159

Galliano, F., Madden, S. C., Jones, A. P., Wilson, C. D., \& Bernard, J. 2005, A\&A, 434, 867

Gaustad, J. E., McCullough, P. R., Rosing, W., \& Van Buren, D. 2001, PASP, 113,1326

Gordon, K. D., Bot, C., Muller, E., et al. 2009, ApJ, 690, L76

Gordon, K. D., Galliano, F., Hony, S., et al. 2010, A\&A, 518, L89

Górski, K. M., Hivon, E., Banday, A. J., et al. 2005, ApJ, 622, 759

Grossi, M., Hunt, L. K., Madden, S., et al. 2010, A\&A, 518, L52

Henize, K. G. 1956, ApJS, 2, 315

Hoang, T., Draine, B. T., \& Lazarian, A. 2010, ApJ, 715, 1462

Hunt, L. K., Dyer, K. K., Thuan, T. X., \& Ulvestad, J. S. 2004, ApJ, 606, 853

Israel, F. P., Wall, W. F., Raban, D., et al. 2010, A\&A, 519, A67

Jarosik, N., Bennett, C. L., Dunkley, J., et al. 2011, ApJS, 192, 14

Kim, S., Staveley-Smith, L., Dopita, M. A., et al. 2003, ApJS, 148, 473

Komatsu, E., Smith, K. M., Dunkley, J., et al. 2011, ApJS, 192, 18

Leroy, A., Bolatto, A., Stanimirovic, S., et al. 2007, ApJ, 658, 1027

Leroy, A. K., Bolatto, A., Bot, C., et al. 2009, ApJ, 702, 352

Lisenfeld, U., Sievers, A., Israel, F., \& Stil, J. 2001, Astrophys. Space Sci. Suppl., 277,105

Madden, S. C. 2000, New Astron. Rev., 44, 249

Madden, S. C., Poglitsch, A., Geis, N., Stacey, G. J., \& Townes, C. H. 1997, ApJ, 483, 200

Meixner, M., Gordon, K. D., Indebetouw, R., et al. 2006, AJ, 132, 2268

Meixner, M., Galliano, F., Hony, S., et al. 2010, A\&A, 518, L71

Mennella, A., Bersanelli, M., Butler, R. C., et al. 2011, A\&A, 536, A3

Meny, C., Gromov, V., Boudet, N., et al. 2007, A\&A, 468, 171

Miville-Deschênes, M., \& Lagache, G. 2005, ApJS, 157, 302

Mizuno, N., Rubio, M., Mizuno, A., et al. 2001, PASJ, 53, L45

Muller, E., Staveley-Smith, L., Zealey, W., \& Stanimirović, S. 2003, MNRAS, 339,105

O'Halloran, B., Madden, S. C., \& Abel, N. P. 2008, ApJ, 681, 1205

O'Halloran, B., Galametz, M., Madden, S. C., et al. 2010, A\&A, 518, L58

Paradis, D. 2007, Ph.D. Thesis

Paradis, D., Bernard, J., \& Mény, C. 2009, A\&A, 506, 745

Paradis, D., Veneziani, M., Noriega-Crespo, A., et al. 2010, A\&A, 520, L8

Planck Collaboration 2011a, A\&A, 536, A1

Planck Collaboration 2011b, A\&A, 536, A2

Planck Collaboration 2011c, A\&A, 536, A7

Planck Collaboration 2011d, A\&A, 536, A8

Planck Collaboration 2011e, A\&A, 536, A9

Planck Collaboration 2011f, A\&A, 536, A10

Planck Collaboration 2011g, A\&A, 536, A11

Planck Collaboration 2011h, A\&A, 536, A12

Planck Collaboration 2011i, A\&A, 536, A13

Planck Collaboration 2011j, A\&A, 536, A14

Planck Collaboration 2011k, A\&A, 536, A15

Planck Collaboration 20111, A\&A, 536, A16

Planck Collaboration 2011m, A\&A, 536, A17 
Planck Collaboration 2011n, A\&A, 536, A18 Planck Collaboration 20110, A\&A, 536, A19 Planck Collaboration 2011p, A\&A, 536, A20 Planck Collaboration 2011q, A\&A, 536, A21 Planck Collaboration 2011r, A\&A, 536, A22 Planck Collaboration 2011s, A\&A, 536, A23 Planck Collaboration 2011t, A\&A, 536, A24 Planck Collaboration 2011u, A\&A, 536, A25

Planck Collaboration 2011v, The Explanatory Supplement to the Planck Early Release Compact Source Catalogue (ESA) Planck Collaboration 2011w, A\&A, 536, A26 Planck HFI Core Team 2011a, A\&A, 536, A4 Planck HFI Core Team 2011b, A\&A, 536, A6 Poglitsch, A., Krabbe, A., Madden, S. C., et al. 1995, ApJ, 454, 293

Reach, W. T., Dwek, E., Fixsen, D. J., et al. 1995, ApJ, 451, 188

Roman-Duval, J., Israel, F. P., Bolatto, A., et al. 2010, A\&A, 518, L74

Rowan-Robinson, M. 1992, MNRAS, 258, 787

Sandstrom, K. M., Bolatto, A. D., Draine, B. T., Bot, C., \& Stanimirović, S. 2010, ApJ, 715, 701

Santini, P., Maiolino, R., Magnelli, B., et al. 2010, A\&A, 518, L154

Serra Díaz-Cano, L., \& Jones, A. P. 2008, A\&A, 492, 127

Shetty, R., Kauffmann, J., Schnee, S., \& Goodman, A. A. 2009a, ApJ, 696, 676

Shetty, R., Kauffmann, J., Schnee, S., Goodman, A. A., \& Ercolano, B. 2009b, ApJ, 696, 2234

Silsbee, K., Ali-Haimoud, Y., \& Hirata, C. M. 2011, MNRAS, 411, 2750

Stanimirovic, S., Staveley-Smith, L., Dickey, J. M., Sault, R. J., \& Snowden, S. L. 1999, MNRAS, 302, 417

Staveley-Smith, L., Sault, R. J., Hatzidimitriou, D., Kesteven, M. J., \& McConnell, D. 1997, MNRAS, 289, 225

Staveley-Smith, L., Kim, S., Calabretta, M. R., Haynes, R. F., \& Kesteven, M. J. 2003, MNRAS, 339, 87

Ysard, N., \& Verstraete, L. 2010, A\&A, 509, A12

Ysard, N., Miville-Deschênes, M. A., \& Verstraete, L. 2010, A\&A, 509, L1

Zacchei, A., Maino, D., Baccigalupi, C., et al. 2011, A\&A, 536, A5

Zhu, M., Papadopoulos, P. P., Xilouris, E. M., Kuno, N., \& Lisenfeld, U. 2009, ApJ, 706, 941

1 Aalto University Metsähovi Radio Observatory, Metsähovintie 114, 02540 Kylmälä, Finland

2 Agenzia Spaziale Italiana Science Data Center, c/o ESRIN, via Galileo Galilei, Frascati, Italy

3 Astroparticule et Cosmologie, CNRS (UMR7164), Université Denis Diderot Paris 7, Bâtiment Condorcet, 10 rue A. Domon et Léonie Duquet, Paris, France

4 Astrophysics Group, Cavendish Laboratory, University of Cambridge, J J Thomson Avenue, Cambridge CB3 OHE, UK

5 Atacama Large Millimeter/submillimeter Array, ALMA Santiago Central Offices, Alonso de Cordova 3107, Vitacura, Casilla 763 0355, Santiago, Chile

${ }^{6}$ CITA, University of Toronto, 60 St. George St., Toronto, ON M5S 3H8, Canada

7 CNRS, IRAP, 9 Av. colonel Roche, BP 44346, 31028 Toulouse Cedex 4, France e-mail: Jean-Philippe. Bernard@cesr. fr

8 California Institute of Technology, Pasadena, California, USA

9 DAMTP, University of Cambridge, Centre for Mathematical Sciences, Wilberforce Road, Cambridge CB3 0WA, UK

10 DSM/Irfu/SPP, CEA-Saclay, 91191 Gif-sur-Yvette Cedex, France

11 DTU Space, National Space Institute, Juliane Mariesvej 30, Copenhagen, Denmark

12 Departamento de Física, Universidad de Oviedo, Avda. Calvo Sotelo $\mathrm{s} / \mathrm{n}$, Oviedo, Spain

13 Department of Astronomy and Astrophysics, University of Toronto, 50 Saint George Street, Toronto, Ontario, Canada

14 Department of Astronomy and Earth Sciences, Tokyo Gakugei University, Koganei, Tokyo 184-8501, Japan

15 Department of Physical Science, Graduate School of Science, Osaka Prefecture University, 1-1 Gakuen-cho, Naka-ku, Sakai, Osaka 5998531, Japan

16 Department of Physics \& Astronomy, University of British Columbia, 6224 Agricultural Road, Vancouver, British Columbia, Canada
17 Department of Physics, Gustaf Hällströmin katu 2a, University of Helsinki, Helsinki, Finland

18 Department of Physics, Nagoya University, Chikusa-ku, Nagoya, 464-8602, Japan

19 Department of Physics, Princeton University, Princeton, New Jersey, USA

20 Department of Physics, Purdue University, 525 Northwestern Avenue, West Lafayette, Indiana, USA

21 Department of Physics, University of California, Berkeley, California, USA

22 Department of Physics, University of California, One Shields Avenue, Davis, California, USA

23 Department of Physics, University of California, Santa Barbara, California, USA

${ }^{24}$ Department of Physics, University of Illinois at Urbana-Champaign, 1110 West Green Street, Urbana, Illinois, USA

25 Dipartimento di Fisica G. Galilei, Università degli Studi di Padova, via Marzolo 8, 35131 Padova, Italy

26 Dipartimento di Fisica, Università La Sapienza, P. le A. Moro 2, Roma, Italy

27 Dipartimento di Fisica, Università degli Studi di Milano, via Celoria, 16 , Milano, Italy

28 Dipartimento di Fisica, Università degli Studi di Trieste, via A. Valerio 2, Trieste, Italy

29 Dipartimento di Fisica, Università di Ferrara, Via Saragat 1, 44122 Ferrara, Italy

30 Dipartimento di Fisica, Università di Roma Tor Vergata, via della Ricerca Scientifica, 1, Roma, Italy

31 Discovery Center, Niels Bohr Institute, Blegdamsvej 17, Copenhagen, Denmark

32 Dpto. Astrofísica, Universidad de La Laguna (ULL), 38206 La Laguna, Tenerife, Spain

33 European Southern Observatory, ESO Vitacura, Alonso de Cordova 3107, Vitacura, Casilla 19001, Santiago, Chile

34 European Space Agency, ESAC, Planck Science Office, Camino bajo del Castillo, s/n, Urbanización Villafranca del Castillo, Villanueva de la Cañada, Madrid, Spain

35 European Space Agency, ESTEC, Keplerlaan 1, 2201 AZ Noordwijk, The Netherlands

${ }^{36}$ Helsinki Institute of Physics, Gustaf Hällströmin katu 2, University of Helsinki, Helsinki, Finland

37 INAF - Osservatorio Astrofisico di Catania, via S. Sofia 78, Catania, Italy

38 INAF - Osservatorio Astronomico di Padova, vicolo dell'Osservatorio 5, Padova, Italy

39 INAF - Osservatorio Astronomico di Roma, via di Frascati 33, Monte Porzio Catone, Italy

40 INAF - Osservatorio Astronomico di Trieste, via G.B. Tiepolo 11, Trieste, Italy

41 INAF/IASF Bologna, via Gobetti 101, Bologna, Italy

42 INAF/IASF Milano, via E. Bassini 15, Milano, Italy

43 INRIA, Laboratoire de Recherche en Informatique, Université Paris-Sud 11, Bâtiment 490, 91405 Orsay Cedex, France

44 IPAG: Institut de Planétologie et d'Astrophysique de Grenoble, Université Joseph Fourier, Grenoble 1/CNRS-INSU, UMR 5274, 38041 Grenoble, France

45 Imperial College London, Astrophysics group, Blackett Laboratory, Prince Consort Road, London, SW7 2AZ, UK

46 Infrared Processing and Analysis Center, California Institute of Technology, Pasadena, CA 91125, USA

47 Institut Néel, CNRS, Université Joseph Fourier Grenoble I, 25 rue des Martyrs, Grenoble, France

48 Institut d'Astrophysique Spatiale, CNRS (UMR8617) Université Paris-Sud 11, Bâtiment 121, Orsay, France

49 Institut d'Astrophysique de Paris, CNRS UMR7095, Université Pierre \& Marie Curie, 98bis boulevard Arago, Paris, France

50 Institute of Astronomy and Astrophysics, Academia Sinica, Taipei, Taiwan 
51 Institute of Astronomy, University of Cambridge, Madingley Road, Cambridge CB3 0HA, UK

52 Institute of Theoretical Astrophysics, University of Oslo, Blindern, Oslo, Norway

53 Instituto de Astrofísica de Canarias, C/Vía Láctea s/n, La Laguna, Tenerife, Spain

54 Instituto de Física de Cantabria (CSIC-Universidad de Cantabria), Avda. de los Castros s/n, Santander, Spain

55 Jet Propulsion Laboratory, California Institute of Technology, 4800 Oak Grove Drive, Pasadena, California, USA

56 Jodrell Bank Centre for Astrophysics, Alan Turing Building, School of Physics and Astronomy, The University of Manchester, Oxford Road, Manchester, M13 9PL, UK

57 Kavli Institute for Cosmology Cambridge, Madingley Road, Cambridge, CB3 OHA, UK

58 LERMA, CNRS, Observatoire de Paris, 61 avenue de l'Observatoire, Paris, France

59 Laboratoire AIM, IRFU/Service d'Astrophysique - CEA/DSM CNRS - Université Paris Diderot, Bât. 709, CEA-Saclay, 91191 Gifsur-Yvette Cedex, France

${ }^{60}$ Laboratoire Traitement et Communication de l'Information, CNRS (UMR 5141) and Télécom ParisTech, 46 rue Barrault, 75634 Paris Cedex 13, France

${ }^{61}$ Laboratoire de Physique Subatomique et de Cosmologie, CNRS/IN2P3, Université Joseph Fourier Grenoble I, Institut National Polytechnique de Grenoble, 53 rue des Martyrs, 38026 Grenoble Cedex, France

${ }^{62}$ Laboratoire de l'Accélérateur Linéaire, Université Paris-Sud 11, CNRS/IN2P3, Orsay, France

${ }^{63}$ Lawrence Berkeley National Laboratory, Berkeley, California, USA

${ }^{64}$ Max-Planck-Institut für Astrophysik, Karl-Schwarzschild-Str. 1, 85741 Garching, Germany
65 MilliLab, VTT Technical Research Centre of Finland, Tietotie 3, Espoo, Finland

66 National University of Ireland, Department of Experimental Physics, Maynooth, Co. Kildare, Ireland

67 Niels Bohr Institute, Blegdamsvej 17, Copenhagen, Denmark

68 Observational Cosmology, Mail Stop 367-17, California Institute of Technology, Pasadena, CA, 91125, USA

69 Observatoire Astronomique de Strasbourg, CNRS, UMR 7550, 67000 Strasbourg, France

70 Optical Science Laboratory, University College London, Gower Street, London, UK

71 SISSA, Astrophysics Sector, via Bonomea 265, 34136, Trieste, Italy

72 SUPA, Institute for Astronomy, University of Edinburgh, Royal Observatory, Blackford Hill, Edinburgh EH9 3HJ, UK

73 School of Physics and Astronomy, Cardiff University, Queens Buildings, The Parade, Cardiff, CF24 3AA, UK

74 Space Sciences Laboratory, University of California, Berkeley, California, USA

75 Spitzer Science Center, 1200 E. California Blvd., Pasadena, California, USA

76 Stanford University, Dept of Physics, Varian Physics Bldg, 382 via Pueblo Mall, Stanford, California, USA

77 Université de Toulouse, UPS-OMP, IRAP, 31028 Toulouse Cedex 4, France

78 Universities Space Research Association, Stratospheric Observatory for Infrared Astronomy, MS 211-3, Moffett Field, CA 94035, USA

79 University of Granada, Departamento de Física Teórica y del Cosmos, Facultad de Ciencias, Granada, Spain

${ }^{80}$ University of Miami, Knight Physics Building, 1320 Campo Sano Dr., Coral Gables, Florida, USA

81 Warsaw University Observatory, Aleje Ujazdowskie 4, 00-478 Warszawa, Poland 